Estabilidade de sistemas detetáveis com custo médio a longo prazo limitado

\author{
Brenno Gustavo Barbosa
}



Data de Depósito:

Assinatura:

\title{
Estabilidade de sistemas detetáveis com custo médio a longo prazo limitado
}

\author{
Brenno Gustavo Barbosa
}

Orientador: Prof. Dr. Eduardo Fontoura Costa

Dissertação apresentada ao Instituto de Ciências Matemáticas e de Computação - ICMC-USP, como parte dos requisitos para obtenção do título de Mestre em Ciências - Ciências de Computação e Matemática Computacional. EXEMPLAR DE DEFESA

USP - São Carlos

Março de 2012 
Ficha catalográfica elaborada pela Biblioteca Prof. Achille Bassi e Seção Técnica de Informática, ICMC/USP, com os dados fornecidos pelo(a) autor(a)

B228e

Barbosa, Brenno Gustavo

Estabilidade de Sistemas Detetáveis com Custo Médio a Longo Prazo Limitado / Brenno Gustavo

Barbosa; orientador Eduardo Fontoura Costa. -- São Carlos, 2012.

$55 \mathrm{p}$.

Dissertação (Mestrado - Programa de Pós-Graduação en Ciências de Computação e Matemática Computacional) -Instituto de Ciências Matemáticas e de Computação, Universidade de Säo Paulo, 2012.

1. Sistemas lineares com saltos markovianos. 2 . Sistemas dinâmicos generalizados. 3. Custo médio a longo prazo. 4. Detetabilidade fraca. I. Costa, Eduardo Fontoura, orient. II. Título. 


\section{Agradecimento}

Não há como expressar em palavras o que as ações de todos que listo aqui representam, mas espero que possa deixar registrado uma pequena, mas verdadeira, prova de meu reconhecimento a todos.

A meus pais, Domingos e Tania, simplesmente por tudo.

A meus avós, Alaor e Luzia, verdadeiros motivos de admiração e inspiração que sempre estiveram ao meu lado.

A meus amigos de longa data Thiago, Jaqueline Brito e Jaqueline de Matos, que com sua amizade me deram força para prosseguir com o trabalho, tornando muito divertido na realidade.

Ao Professor Eduardo, que com sua dedicação me ensinou muito mais do que qualquer livro ou curso poderia me ensinar.

À FAPESP - Fundação de Amparo a Pesquisa do Estado de São Paulo - pelo suporte financeiro.

A todos, meus mais sinceros agradecimentos. 



\section{Resumo}

Neste trabalho estudamos a estabilidade assintótica de Lagrange para duas classes de sistemas, sob as hipóteses de detetabilidade fraca e de limitação do custo médio a longo prazo. Para sistemas lineares com saltos markovianos com ruído aditivo, a equivalência entre estabilidade e as condições mencionadas será provada. Para sistemas dinâmicos generalizados, provaremos a estabilidade sob uma condição adicional.

Palavras-chave: Sistemas lineares com saltos markovianos, sistemas dinâmicos generalizados, custo médio a longo prazo, detetabilidade fraca. 



\section{Abstract}

In this work we study Lagrange asymptotic stability for two classes of systems, under conditions of weak detectability and boundedness of the long run average cost. For Markov jump linear systems with additive noise, the equivalence between stability and the aforementioned conditions is proved. For generalized dynamical systems, we prove stability under an additional condition.

Keywords: Markov jump linear systems, generalized dynamical systems, long run average cost, weak detectability. 



\section{Sumário}

\begin{tabular}{ll}
\hline Lista de Figuras & 13
\end{tabular}

\begin{tabular}{ll}
\hline Lista de Tabelas & 15
\end{tabular}

\begin{tabular}{lll}
\hline & Introdução & 17
\end{tabular}

1.1 Sobre o texto . . . . . . . . . . . . . . . . . . . . . . . . . 18

$1.1 .1 \quad$ Conteúdo e estrutura . . . . . . . . . . . . . . . . . . . . . . . . . . 18

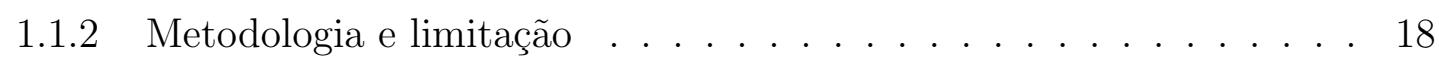

1.2 Comentários sobre bibliografia $\ldots \ldots \ldots \ldots$

2 Definições e Resultados Preliminares $\quad 21$

2.1 Sistemas Lineares com Saltos Markovianos . . . . . . . . . . . . . . . . . . 21

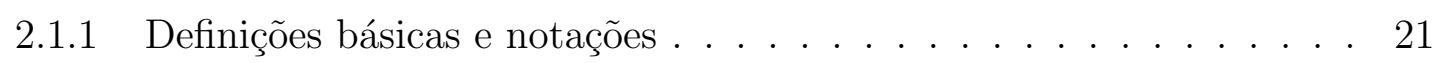

2.1 .2 Estabilidade . . . . . . . . . . . . . . . . . . . . 25

2.1 .3 Cones $\ldots \ldots \ldots \ldots \ldots$

2.1 .4 Custo médio a longo prazo e Detetabilidade . . . . . . . . . . . . . 31

2.2 Sistemas dinâmicos generalizados $\ldots \ldots \ldots$. . . . . . . . . . . 35

3 Detetabilidade e custo médio a longo prazo e estabilidade 39

3.1 Sistemas Lineares com Saltos Markovianos . . . . . . . . . . . . . . . . . . 39

3.1 .1 Teoremas principais . . . . . . . . . . . . . . . . . . . . . . . . . . . . . . . . . . . . . . .

$3.1 .2 \quad$ Exemplos Numéricos $\ldots \ldots \ldots$. . . . . . . . . . . . . . . . 42

3.2 Sistemas dinâmicos generalizados . . . . . . . . . . . . . . . . . . . . 45

3.2 .1 Teorema Principal . . . . . . . . . . . . . . . . . . . . . . 45

3.2 .2 Um resultado sobre estabilidade exponencial . . . . . . . . . . . . . 49

\begin{tabular}{lll}
\hline Conclusões & 51
\end{tabular}

\begin{tabular}{ll}
\hline Referências Bibliográficas & 53
\end{tabular}

\begin{tabular}{ll}
\hline A Apêndice & 57
\end{tabular}

A.1 Cadeias de Markov . . . . . . . . . . . . . . . . . . . . . . . . . . . . . 57

A.2 Alguns resultados em Álgebra linear e séries . . . . . . . . . . . . . . . . 58 
A.3 Testes de estabilidade e detetabilidade para sistemas lineares com saltos Markovianos . . . . . . . . . . . . . . . . . 6 60 


\section{Lista de Figuras}

3.1 Custo $J(t, X)$ para 5 valores diferentes de $X$ (tracejado), a reta $t / 3$ (sólida) e os correspondentes valores de $t_{0}$, para o sistema no Caso 1. . . . . . . . . 43

3.2 Custo $J(t, X)$ em escala logarítmica para 10 valores diferentes de $X$, para o sistema no Caso 2 . . . . . . . . . . . . . . . . . . . . . 43

3.3 Custo $J(t, X)$ para diferentes valores de $X$, para o sistema no Caso 3. . . . 44 



\section{Lista de Tabelas}

3.1 Relação entre detetabilidade e estabilidade assintótica no sentido de Lagrange para diferentes valores de $\alpha$ e $\beta$. . . . . . . . . . . . . . . . 42 



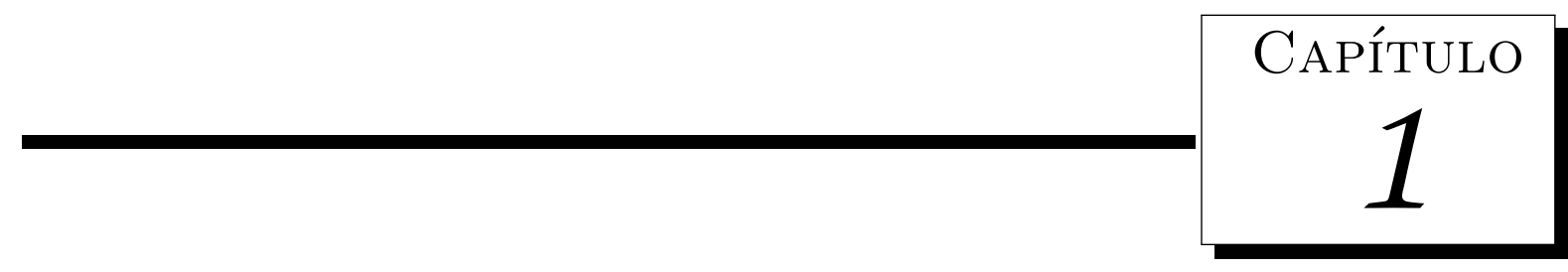

\section{Introdução}

Plagiarize, Let no one else's work evade your eyes, Remember why the good Lord made your eyes,

So don't shade your eyes, But plagiarize, plagiarize, plagiarize Only be sure always to call it please 'research'

Tom Lehrer

O uso de controle de sistemas existe há centenas talvez milhares de anos, mas apenas no fim do século XIX começou-se a utilizar sistematicamente a matemática na modelagem de sistemas. Foi possível apreciar a utilidade da matemática nesta área através do projeto de sistemas que apresentavam características de interesse. Uma das principais características de interesse em um sistema é com certeza estabilidade. Porém existem muitos conceitos diferentes de estabilidade, por exemplo, a capacidade de um sistema voltar ao seu estado ideal de operação após ser perturbado, ou a capacidade de um sistema em manter limitadas suas características de forma a manter-se em condições de operação.

Dentro deste último exemplo há uma classe peculiar de sistemas que são aqueles capazes de suportar uma sobrecarga inicial, mas que ao continuar em estado de tensão elevada eventualmente sucumbem e ficam inoperantes para qual seja sua função.

Para o bom funcionamento destes sistemas, deve haver um instante futuro no qual o sistema não está mais em situação crítica e é este tipo de estabilidade que estudaremos aqui, a chamada estabilidade assintótica no sentido Lagrange.

O objetivo deste trabalho é obter condições suficientes para que ocorra estabilidade como descrita acima. Dentre os candidatos a condição suficiente notamos que a limitação do chamado custo médio a longo prazo e a detetabilidade fraca agem de forma a privilegiar a estabilidade e aparentemente cumprem o papel que esperamos. E é neste ponto que começa o trabalho. 


\subsection{Sobre o texto}

\subsubsection{Conteúdo e estrutura}

Neste trabalho trataremos de duas classes de sistemas: sistemas lineares com saltos markovianos e sistemas dinâmicos generalizados. Em relação a primeira classe, nos restringiremos ao caso em que os parâmetros são independentes do tempo. Mostraremos que a limitação do custo médio a longo prazo conjuntamente com detetabilidade fraca são condições necessárias e suficientes para garantir a estabilidade assintótica de Lagrange. Mostraremos também que não há como tornar mais fraca ou mais forte a afirmação, no seguinte sentido: não há como remover nem detetabilidade nem custo limitado ou trocá-los por observabilidade e existência do custo. Por fim trataremos uma aplicação com controle de realimentação de saída.

Em relação ao sistemas dinâmicos generalizados vamos mostrar apenas que para uma certa classe de sistemas, que contém vários casos relevantes, uma hipótese de limitação do custo de horizonte finito associado e detetabilidade fraca implicam na estabilidade mencionada. É importante notar que a hipótese sobre o custo implicará na limitação do custo médio a longo prazo. Além disso, mostraremos que em uma condição especial a estabilidade considerada apresenta uma característica mais forte, com decrescimento exponencial do estado.

O trabalho se organiza da seguinte forma: no capítulo 2 apresentamos as definições e alguns resultados preliminares para cada tema de estudo. No capítulo 3 apresentaremos o conteúdo exposto acima. No capítulo final apresentamos as conclusões deste trabalho. Há também um apêndice destinado a complementar certos detalhes de provas ou exemplos.

Cabe ressaltar que este trabalho resultou até o momento em dois trabalhos: o artigo Barbosa e Costa (2012b) e o trabalho submetido Barbosa e Costa (2012a).

\subsubsection{Metodologia e limitação}

A ideia deste trabalho é torná-lo o mais auto contido possível e por isso tentaremos provar a maioria dos resultados auxiliares que talvez sejam de difícil acesso para algum leitor ou que podem não estar na forma como vamos utilizá-los. No caso de sistemas lineares com saltos markovianos vamos empregar o método de operadores já bastante difundido na área. Este método dá origem ao estudo de sistemas positivos e é com base nesta metodologia que obteremos os resultados. Porém, apesar da simplicidade com que estas ferramentas permitem tratar o problema, elas limitam o poder do método e por isso não é possível generalizar com estas armas o resultado para sistemas com parâmetros variando no tempo e esta é a grande limitação que sofremos neste tema. É importante ressaltar que os métodos desenvolvidos no segundo tema foram empregados inicialmente para tratar este problema, mas as hipóteses que teriam que ser feitas não são praticáveis e portanto abandonamos a técnica para este caso.

Em relação ao segundo problema, pouquíssimo instrumental matemático é necessário. A ideia utilizada de obter contradições a partir da limitação do custo é explorada de uma forma um pouco diferente do primeiro caso. De uma forma geral o plano é mostrar que 
existe uma região de estados que o sistema deve ficar, caso contrário há violação do custo. A maior limitação neste caso é que o sistema deve pertencer a uma classe especial. Há também limitações de ordem técnica, por exemplo, não conhecemos técnicas para verificar a condição de detetabilidade fraca empregada.

As contribuições técnicas deste trabalho são basicamente duas: a ideia de utilizar teoria espectral de operadores positivos e uma variação do método empregado em Costa e do Val (2003) no que tange as avaliações necessárias. Acreditamos que tais métodos podem ser utilizados em outras situações para obter resultados de limitação de trajetórias.

\subsection{Comentários sobre bibliografia}

As referências que aparecem no corpo do texto têm intenção de ser material para uso no texto e por isto vamos fazer uma breve revisão bibliográfica do estado atual do tema nesta seção.

Há duas referências mais relevantes e próximas ao tema, ambas dos mesmos autores, Vargas e do Val (2010, 2011). Nestes dois textos são estudadas as relações entre o custo médio a longo prazo, a observabilidade e noções de estabilidade dentre as quais a estabilidade uniforme do segundo momento que muito lembra a estabilidade assintótica no sentido de Lagrange. A segunda destas referências tem um sabor bastante parecido com o que apresentamos para sistemas com saltos, pois é mostrado que observabilidade e custo médio a longo prazo implicam em estabilidade, mas as hipóteses de custo médio são diferentes e os autores tratam de sistemas estocásticos sem saltos. Quanto a noção de detetabilidade alguns artigos que falam sobre a detetabilidade fraca no caso de sistemas estocásticos são Li et al. (2009) que utiliza resultados de Damm (2007) ou Damm e Ethington (2009) sobre testes de detetabilidade. Em relação ao custo médio, há resultados em relação ao problema de otimização do custo para sistemas estáveis, veja Silva e Costa (2009) e resultados sobre limitação do custo por limitantes lineares Costa et al. (2011). 


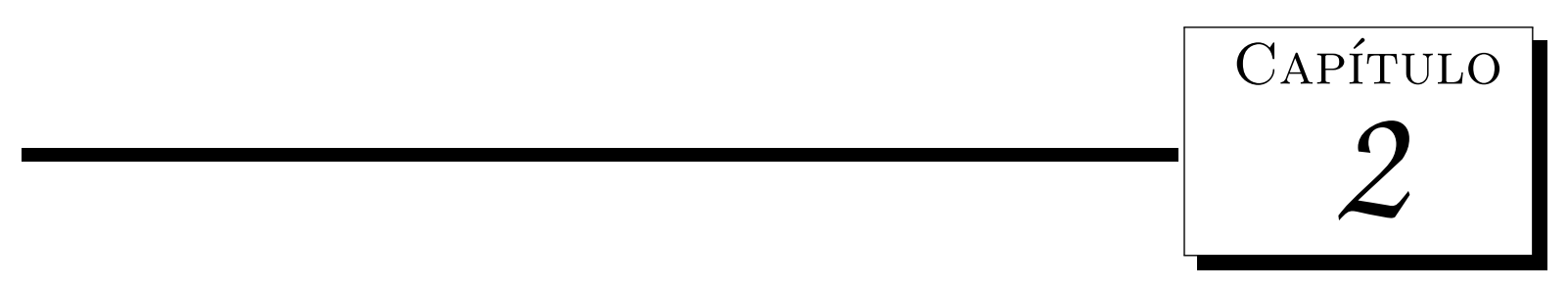

\section{Definições e Resultados Preliminares}

Os dois problemas a serem abordados neste texto tem o tempo definido nos números naturais $\mathbb{N}$ incluindo o zero, o que é habitualmente chamado na literatura de sistemas de controle a tempo discreto não negativo. Denotaremos por $\mathbb{R}$ o conjunto dos números reais e por $\mathbb{R}^{+}$o subconjunto dos números reais não negativos. Além disso, todo espaço vetorial tratado aqui é real, salvo os casos em que for necessário tratar de autovalores complexos. Neste caso consideramos a complexificação do espaço e de operadores lineares. No que segue $\lfloor\cdot\rfloor$ e $\lceil\cdot\rceil$ representam a função menor inteiro e a maior inteiro respectivamente.

A primeira seção deste capítulo tratará de sistemas lineares com saltos markovianos, a segunda seção de sistemas dinâmicos generalizados.

\subsection{Sistemas Lineares com Saltos Markovianos}

Nesta seção vamos apresentar os conceitos e ferramentas necessárias a investigação do problema da estabilidade de sistemas lineares com saltos markovianos detetáveis com custo médio a longo prazo limitado. Ao final desta seção vamos formular o problema de forma mais precisa do que na introdução.

\subsubsection{Definições básicas e notações}

Seja $(\Omega, \mathcal{F}, P)$ um espaço de probabilidade criado de tal forma a comportar a seguinte estrutura especial: dados uma família de variáveis aleatória discretas e com mesma imagem $\theta(k), k \in \mathbb{N}$, uma família de vetores aleatórios de dimensão $r, w(k), k \in \mathbb{N}$ e um vetor aleatório $x(0)$ de dimensão $n$ então

(H1) Para $k \geq 1$ e $t \geq k, \theta(t)$ independe condicionalmente de $\theta(n), n<k-1$, de $w(n), n<k$ e de $x(0)$ dado $\theta(k-1)$.

(H2) Para $k \geq 0, w(k)$ independe de $\theta(t), t \leq k$, de $w(t), t<k$ e de $x(0)$. 
(H3) Para todo $k$ tem-se $E(w(k))=0$ e sem perda de generalidade $E\left(w(k) w(k)^{\prime}\right)=I_{r}$ onde $E$ denota a esperança com respeito à $P$ e $I_{r}$ é a matriz identidade de ordem $r \times r$.

(H4) $x(0)$ tem covariância finita.

A existência/construção de tal espaço de probabilidade não foi considerada neste projeto, por estar além dos propósitos do tema. Nos limitados a trabalhar com este espaço mesmo com consciência de que a não existência do mesmo acarretaria alguns problemas técnicos no decorrer do texto.

Vamos supor que a imagem comum das aplicações $\theta(k)$ é o conjunto $S=\{1,2, \ldots, N\}$. O processo estocástico $\theta(k), k \in \mathbb{N}$ constitui uma cadeia de Markov (vide apêndice seção A.1 do apêndice) com espaço de estado discreto cuja matriz de transição de probabilidade denotaremos por $\mathbf{P}$ com $i$-ésimo elemento da $j$-ésima coluna denotado por $p_{i j}$.

O processo $w(k), k \in \mathbb{N}$ é um ruído branco com matriz de covariância identidade. Este é um dos tipos mais comuns de distúrbios presentes em diversos sistemas. A independência descrita entre $w(k), \theta(k)$ e $x(0)$ é uma forma de dizer que não há uma influência do passado, exceto na cadeia de Markov. O que deve ficar claro é que não é exigida independência entre a variável $x(0)$ e a variável $\theta(0)$ o que traduz que o estado pode ser escolhido dependendo de $\theta(0)$ o que é de grande importância para a teoria subsequente.

O interesse neste modelo probabilístico do ponto de vista teórico ficará claro na demonstração da proposição 1. Utilizando este espaço podemos definir o objeto de estudo desta seção. Considere o sistema estocástico definido pela equação de diferenças estocástica

$$
\left\{\begin{array}{l}
x(t+1)=A_{\theta(k)} x(t)+G_{\theta(k)} w(t), \quad x(0)=x_{0} \\
c(t)=x(t)^{\prime} Q_{\theta(t)} x(t),
\end{array}\right.
$$

com $x(t)$ um vetor aleatório de dimensão $n, A_{i}, G_{i}$ e $Q_{i}$ matrizes de ordem $n \times n, n \times r$ e $n \times n$ para $i \in S$ e $c(t)$ uma variável aleatória. Supomos que $Q_{i}$ é positiva semidefinida para $i \in S$. O sobrescrito ' indica o transposto de uma matriz/vetor. O estado deste sistema é formado pelo par $(x(k), \theta(k))$. O sistema acima pode ser entendido da seguinte forma: no instante de tempo $t$ a variável $x$ evolui segundo um sistema linear associado ao estado da cadeia de Markov.

Os espaços vetoriais $M_{n, m}^{N}$ são espaços formados por N-túplas ordenadas de matrizes com operações de soma e produto por escalar tomado coordenada a coordenada, isto é,

$$
U=\left(U_{1}, \ldots, U_{N}\right), V=\left(V_{1}, \ldots, V_{N}\right) \in M_{n, m}^{N}, \lambda \in \mathbb{R}
$$

então

$$
\begin{aligned}
U+V & =\left(U_{1}+V_{1}, \ldots, U_{N}+V_{N}\right), \\
\lambda U & =\left(\lambda U_{1}, \ldots, \lambda U_{N}\right) .
\end{aligned}
$$

Escreveremos $S_{n}^{N}$ para o subespaço formado por N-túplas de matrizes simétricas e $S_{n}^{+N}$ para o subconjunto de $S_{n}^{N}$ formado por matrizes positivas semidefinidas. Com esta notação 
temos $A \in M_{n, n}^{N}, G \in M_{n, r}^{N}, Q \in S_{n}^{+N}$. Definimos também nestes espaços vetoriais o seguinte produto interno (o que os torna espaços de Hilbert uma vez que tem dimensão finita)

$$
\langle A, B\rangle=\sum_{i=1}^{N} \operatorname{tr}\left(A_{i} B_{i}^{\prime}\right) .
$$

Denotamos por $\|\cdot\|$ a norma associada a este produto, isto é

$$
\|A\|=\sqrt{\langle A, A\rangle} .
$$

Vamos denotar por $\pi(t)$ a distribuição de $\theta(t)$, isto é, $\pi(t)=[P(\theta(t)=1) \cdots P(\theta(t)=N)]$. Introduzimos enfim a coleção de matrizes de segundo momento condicionado pertencentes a $S_{n}^{+N}$ seguindo os textos da área como Costa et al. (2005) [Capítulo 3]

$$
X_{i}(t):=E\left(x(t) x(t)^{\prime} 1_{\{\theta(t)=i\}}\right), \quad i \in S,
$$

onde $1_{A}: \Omega \rightarrow\{0,1\}$ é a função indicadora do evento $A$. Como $\sum_{i=1}^{N} 1_{\{\theta(t)=i\}}=1$ segue que podemos decompor a matriz de covariância de $x(t)$ como, $E\left(x(t) x(t)^{\prime}\right)=\sum_{i=1}^{N} X_{i}(t)$. O que torna interessante em definir estas matrizes é que podemos calcular as matrizes no instante posterior a partir das matrizes presentes e este é o conteúdo da

Proposição 1. A coleção de matrizes de segundo momento condicionado, $X(t)$, satisfaz a seguinte recorrência

$$
X(t+1)=\mathcal{T}(X(t))+\Sigma(t)
$$

onde

$$
\begin{aligned}
\mathcal{T}_{i}(U) & =\sum_{j=1}^{N} p_{j i} A_{j} U_{j} A_{j}^{\prime}, \\
\Sigma_{i}(t) & =\sum_{j=1}^{N} p_{j i} G_{j} G_{j}^{\prime} \pi_{j}(t)
\end{aligned}
$$

A matriz $X(0)$ assume qualquer valor em $S_{n}^{+N}$.

Demonstração. Desenvolvendo $X(t+1)$ obtemos

$$
\begin{aligned}
X_{i}(t+1) & =E\left(x(t+1) x(t+1)^{\prime} 1_{\{\theta(t+1)=i\}}\right) \\
& =E\left(A_{\theta(t)} x(t) x(t)^{\prime} A_{\theta(t)}^{\prime} 1_{\{\theta(t+1)=i\}}\right)+E\left(G_{\theta(t)} w(t) w(t)^{\prime} G_{\theta(t)}^{\prime} 1_{\{\theta(t+1)=i\}}\right)+ \\
& +E\left(G_{\theta(t)} w(t) x(t)^{\prime} A_{\theta(t)}^{\prime} 1_{\{\theta(t+1)=i\}}\right)+E\left(A_{\theta(t)} x(t) w(t)^{\prime} G_{\theta(t)}^{\prime} 1_{\{\theta(t+1)=i\}}\right) .
\end{aligned}
$$

Observe que temos

$$
E\left(A_{\theta(t)} x(t) x(t)^{\prime} A_{\theta(t)}^{\prime} 1_{\{\theta(t+1)=i\}}\right)=\sum_{j=1}^{N} E\left(A_{\theta(t)} x(t) x(t)^{\prime} A_{\theta(t)} 1_{\{\theta(t+1)=i\}} 1_{\{\theta(t)=j\}}\right)
$$

e similarmente para os outros termos de (2.4). Podemos reescrever a equação na forma

$$
E\left(A_{\theta(t)} x(t) x(t)^{\prime} A_{\theta(t)} 1_{\{\theta(t+1)=i\}} 1_{\{\theta(t)=j\}}\right)=A_{j} E\left(x(t) x(t)^{\prime} 1_{\{\theta(t+1)=i\}} 1_{\{\theta(t)=j\}}\right) A_{j}^{\prime} .
$$


Valendo-se do fato que

$$
E\left(x(t) x(t)^{\prime} 1_{\{\theta(t+1)=i\}} 1_{\{\theta(t)=i\}}\right)=E\left(x(t) x(t)^{\prime} 1_{\{\theta(t+1)=i\}} \mid \theta(t)=i\right) P(\theta(t)=i)
$$

e utilizando que $x(t)$ é condicionalmente independente de $1_{\{\theta(t+1)=i\}}$ dado $\theta(t)$ vem que

$$
\begin{aligned}
E\left(x(t) x(t)^{\prime} 1_{\{\theta(t+1)=i\}} \mid \theta(t)=i\right) & =E(x(t) x(t) \mid \theta(t)=j) P(\theta(t)=j) E\left(1_{\{\theta(t+1)=i\}} \mid \theta(t)=j\right) \\
& =X_{j}(t) p_{j i},
\end{aligned}
$$

assim

$$
E\left(A_{\theta(t)} x(t) x(t)^{\prime} A_{\theta(t)}^{\prime} 1_{\{\theta(t+1)=i\}}\right)=\mathcal{T}_{i}(X(k)) .
$$

O segundo termo é trabalhado de forma similar

$$
\begin{aligned}
E\left(G_{\theta(t)} w(t) w(t)^{\prime} G_{\theta(t)}^{\prime} 1_{\{\theta(t+1)=i\}}\right) & =\sum_{j=1}^{N} E\left(G_{\theta(t)} x(t) x(t)^{\prime} G_{\theta(t)} 1_{\{\theta(t+1)=i\}} 1_{\{\theta(t)=j\}}\right) \\
& =\sum_{j=1}^{N} G_{j} E\left(w(k) w(k)^{\prime} 1_{\{\theta(t+1)=i\}} \mid \theta(t)=j\right) P(\theta(t)=i) G_{j}^{\prime}
\end{aligned}
$$

com as hipóteses do espaço de probabilidade

$$
E\left(G_{\theta(t)} w(t) w(t)^{\prime} G_{\theta(t)}^{\prime} 1_{\{\theta(t+1)=i\}}\right)=\Sigma_{i}(t) .
$$

Com argumentos similares se verifica que os dois termos restantes de 2.4 são nulos, então

$$
X(t)=\mathcal{T}(X(t))+\Sigma(t) .
$$

Resta verificar que $X(0)$ pode assumir qualquer valor em $S_{n}^{+N}$. De fato, temos que $E\left(x(0) x(0)^{\prime} 1_{\{\theta(0)=i\}}\right)=E\left(x(0) x(0)^{\prime} \mid \theta(0)=i\right) \pi_{i}(0)$. Sendo que não há restrições sobre a distribuição conjunta de $x(0)$ e $\theta(0)$ é possível que a esperança condicional assuma qualquer matriz positiva semidefinida, o que garante o resultado.

A função $\mathcal{T}$ pode ser estendida para um operador linear em $S_{n}^{N}$ de forma única, cuja forma é exatamente a mesma. Se tivéssemos escolhido $x(0)$ e $\theta(0)$ independentes como é usual na literatura $X(0)$ assumiria valores apenas em um subconjunto próprio de $S_{n}^{+N}$. Completando as notações escrevemos $X(t, X)$ para representar $X(t)$ com condição inicial $X(0)=X$ e observe que

$$
X(t, X)=\mathcal{T}^{t}(X)+\sum_{i=0}^{t-1} \mathcal{T}^{i}\left(\Sigma_{t-1-i}\right) .
$$

Para especificarmos o sistema com o qual estamos trabalhando vamos utilizar a notação $(A, G, Q, \mathbf{P})$. Com esta notação $(A, 0, Q, \mathbf{P})$ é o sistema $(A, G, Q, \mathbf{P})$ sem ruído que é chamado de sistema homogêneo associado a $(A, G, Q, \mathbf{P})$. Quando um item desta notação não for importante ele será omitido da seguinte forma $(A, G, \cdot, \mathbf{P})$ indicando que ele pode ser substituído por qualquer outro sem perda de generalidade da afirmação.

Como última observação antes de prosseguirmos, note que o ruído ter covariância igual a identidade não reduz a generalidade uma vez que estamos multiplicando o ruído por matrizes $G$ que modificam a covariância conforme necessário.

Nosso próximo passo é descrever o tipo de estabilidade que esperamos utilizar neste trabalho. 


\subsubsection{Estabilidade}

Nesta seção vamos definir os três tipos de estabilidade que desempenharam algum papel na seção de sistemas lineares com saltos markovianos. As duas primeiras definições que vamos dar seguem a monografia de (Costa et al., 2005, Capítulo 3) com algumas modificações. Para um estudo mais amplo sobre estabilidade de sistemas estocásticos o leitor pode consultar Kozin (1969). Começaremos definindo a estabilidade em média quadrática que mais precisamente é uma estabilidade assintótica ao ponto 0. Está noção será definida apenas para o sistema homogêneo uma vez que a presença do ruído pode fazer com que o ponto 0 não seja de equilíbrio.

Definição 1. O sistema $(A, 0, \cdot, \mathbf{P})$ é dito estável em média quadrática se

$$
\lim _{t \rightarrow \infty} E\left(x(t)^{\prime} x(t)\right)=0
$$

para todo $x(0)$ e $\theta(0)$.

A próxima forma de estabilidade que também será definida somente para o sistema homogêneo é a estabilidade exponencial do segundo momento.

Definição 2. Dizemos que $(A, 0, \cdot, \mathbf{P})$ é exponencialmente estável em média quadrática se existem $\beta$ e $\xi \in[0,1)$ tais que

$$
E\left(x(t)^{\prime} x(t)\right) \leq \beta \xi^{t} E\left(x(0)^{\prime} x(0)\right) .
$$

As duas noções de estabilidade anteriores desempenham um papel auxiliar. A definição seguinte é o ponto central deste estudo.

Definição 3 (Estabilidade assintótica no sentido de Lagrange). Dizemos que o sistema $(A, G, \cdot P)$ é assintoticamente estável no sentido de Lagrange (em média quadrática) se existe $C$ e para cada $x(0)$ e $\theta(0)$ existe to tal que

$$
E\left(x(t)^{\prime} x(t)\right)<C
$$

sempre que $t>t_{0}$.

Esta definição foi adaptada do livro (Liao et al., 2007, Seção 5.4), onde é fornecida para sistemas determinísticos. Na literatura é mais comum a definição conhecida como sistema uniformemente limitado em instantes futuros (uniformly ultimately bounded), veja (Michel et al., 2001, Definição 3.2.10), (Michel et al., 2008, Definição 3.1.14). No entanto esta definição supõe não uma dependência de $t_{0} \operatorname{com} X(0)$, mas de $t_{0} \operatorname{com}\|X(0)\|$ da seguinte forma, todo $X(0)$ tal que $\|X(0)\|<\alpha$ compartilham o mesmo $t_{0}$. O que motiva o estudo desta forma de estabilidade para a classe de sistemas que estamos considerando é esta definição supõe apenas atratividade para uma bola centrada em 0 ao invés de exigir qual a forma desta bola, como por exemplo exigir que seja um ponto em especial.

A próxima observação mostra que o estudo da estabilidade do sistema (2.1) sem ruído é equivalente ao estudo da estabilidade do sistema (1) associado.

Proposição 2. O sistema 
- $(A, 0, \cdot, \mathbf{P})$ é estável na média quadrática se e somente se

$$
\lim _{t \rightarrow \infty} X(t, X)=0
$$

para todo $X \in S_{n}^{+N}$.

- $(A, 0, \cdot, \mathbf{P})$ é exponencialmente estável na média quadrática se e somente se existem $\bar{\beta}>0$ e $0 \leq \bar{\xi}<1$ tais que

$$
\|X(t, X)\|<\bar{\beta} \bar{\xi}^{t}\|X\|
$$

para todo $X \in S_{n}^{+N}$.

- $(A, G, \cdot, \mathbf{P})$ é assintoticamente estável no sentido de Lagrange se existe $\bar{C}$ e para todo $X \in S_{n}^{+N}$ existe $t_{0}$ tal que

$$
\|X(t, X)\|<\bar{C}
$$

quando $t>t_{0}$.

Demonstração. Isto decorre do fato que $\|X\| \leq\left\|X^{\frac{1}{2}}\right\|^{2} \leq \sqrt{N n}\|X\|$ para todo $X \in$ $S_{n}^{+N}$, onde definimos $X^{\frac{1}{2}}=\left(X_{1}^{\frac{1}{2}}, \ldots, X_{N}^{\frac{1}{2}}\right), X_{i}^{\frac{1}{2}}$ raiz quadrada de $X_{i}$, e $E\left(x(k)^{\prime} x(k)\right)=$ $\sum_{i=1}^{N} \operatorname{tr}\left(X_{i}(k)\right)=\left\|X(k)^{\frac{1}{2}}\right\|^{2}$. Para verificar esta desigualdade basta notar que a norma considerada $\|\cdot\|$ é a soma dos autovalores de cada matriz em $X$ ao quadrado e que vale a desigualdade $\sqrt{a_{1}^{2}+\ldots+a_{n N}^{2}} \leq a_{1}+\ldots+a_{n N} \leq N n \sqrt{a_{1}^{2}+\ldots+a_{n N}^{2}}$ vale para $a_{i} \geq 0$. Vamos verificar o segundo item. Suponha que o sistema seja exponencialmente estável na média quadrática. Temos que

$$
\|X(t)\| \leq E\left(x(t)^{\prime} x(t)\right) \leq \beta \xi^{t} E\left(x(0)^{\prime} x(0)\right) \leq \beta \sqrt{N n} \xi^{t}\|X(0)\|,
$$

fazendo $\bar{\xi}=\xi$ e $\bar{\beta}=\beta \sqrt{N n}$, obtemos o resultado. Os demais seguem analogamente.

O próximo teorema fornece a equivalência entre estabilidade em média quadrática, estabilidade exponencial e uma condição sobre o raio espectral de $\mathcal{T}$. Denotaremos o raio espectral de um operador $T$ por $\rho(T)$. Este resultado foi retirado de (Costa et al. 2005 , Teorema 3.9) e adaptado as necessidades do texto.

Teorema 1 (Estabilidade em média quadrática). São equivalentes as afirmações:

(i) o sistema $(A, 0, \cdot \mathbf{P})$ é estável na média quadrática;

(ii) o raio espectral de $\mathcal{T}$ é menor que 1 ;

(iii) o sistema $(A, 0, \cdot, \mathbf{P})$ é exponencialmente estável na média quadrática.

Demonstração. (i) $\Rightarrow$ (ii). Na próxima seção, proposição 4, veremos que qualquer elemento de $S_{n}^{N}$ podes ser escrito como a diferença de dois elementos de $S_{n}^{+N}$, isto é

$$
S=M-N \quad M, N \in S_{n}^{+N} .
$$


Para todo $S \in S_{n}^{N}$ vale que

$$
\mathcal{T}^{t}(S)=\mathcal{T}^{t}(M)-\mathcal{T}^{t}(N) \quad M, N \in S_{n}^{+N},
$$

como $\lim _{t \rightarrow \infty} \mathcal{T}^{t}(M)=\lim _{t \rightarrow \infty} \mathcal{T}^{t}(N)=0$ então $\lim _{t \rightarrow \infty} \mathcal{T}^{t}(S)=0$. Este último resultado nos diz que se para as condições iniciais em $S_{n}^{+N}$ a evolução segundo o operador $\mathcal{T}$ é estável então ela é estável em todo espaço vetorial $S_{n}^{N}$. Um resultado bem conhecido da teoria de sistemas lineares nos diz que em um espaço vetorial de dimensão vetorial as iteradas de um operador linear convergem a zero se e somente se o raio espectral deste operador é menor que 1. Vamos provar a implicação que necessitamos para manter o texto o mais independente possível. Seja $X$ autovetor com respectivo autovalor $\lambda$ associado a $\rho(\mathcal{T})$, isto é $\rho(T)=|\lambda|$ onde $|\cdot|$ é o módulo de um número complexo. Temos que

$$
\left\|\mathcal{T}^{t}(X)\right\|=\rho(\mathcal{T})^{t}\|X\|
$$

portanto temos que $\rho(\mathcal{T})^{t}$ tende a zero e por isto $\rho(\mathcal{T})<1$.

(ii) $\Rightarrow$ (iii).O próximo passo consiste em mostrar que se o raio espectral do operador $\mathcal{T}$ é menor que 1 então $X(t, X)$ satisfaz a condição necessária para estabilidade exponencial em média quadrática como na proposição 2. Vamos utilizar o lema 10 do apêndice. Como $\rho(\mathcal{T})<1$ existe $\epsilon>0$ suficientemente próximo de zero tal que $\rho(\mathcal{T})+\epsilon<1$. Seja $\xi=\rho(\mathcal{T})+\epsilon$. Temos que existe $t_{0}$ tal que para $t>t_{0}$ vale

$$
\left\|\mathcal{T}^{t}\right\| \leq \xi^{t}
$$

onde $\|\mathcal{T}\|$ é a norma induzida do operador $\mathcal{T}$, definida como $\|\mathcal{T}\|:=\sup \{\|\mathcal{T}(X)\|$ : $\|X\|=1\}$ que satisfaz $\|\mathcal{T}(X)\|=\|\mathcal{T}\|\|X\|$. Tome $\beta:=\max \left(\max _{0 \leq t \leq t_{0}}\left(\frac{\left\|\mathcal{T}^{t}\right\|}{\xi^{t}}\right), 1\right)$. Temos que $\left\|\mathcal{T}^{t}\right\| \leq \beta \xi^{t}$, logo

$$
\left\|\mathcal{T}^{t}(X)\right\| \leq \beta \xi^{t}\|X\|
$$

para todo $X \in S_{n}^{N}$, em particular para $X \in S_{n}^{+N}$.

(iii) $\Rightarrow$ (i). Basta observar que $\left\|\mathcal{T}^{t}(X)\right\| \leq \beta \xi^{t}\|X\|$ implica que $\left\|\mathcal{T}^{t}(X)\right\|$ tende a zero.

A subseção seguinte é um pequeno interlúdio sobre cones em espaços vetoriais com a finalidade de analisar o cone $S_{n}^{+N}$.

\subsubsection{Cones}

Cones são estruturas que geram relações de ordem em espaços vetoriais. A literatura é bastante vasta sobre cones, alguns exemplos são Aliprantis e Tourky (2007); Schaefer e Wolff (1999) sobre cones e ordem, Aliprantis e Burkinshaw (2006); Abramovich e Aliprantis (2002) sobre operadores positivos e Krasnoselskii et al. (1989) sobre aplicações em sistema. Vamos seguir as definições de (Deimling, 1985, Capítulo 6),(Drăgan et al., 2009 , Capítulo 2) com ligeiras modificações.

Definição 4. Um cone convexo em um espaço vetorial $V$ é um conjunto $\mathcal{C} \subset V$ tal que

(i) $s e u, v \in \mathcal{C}$ então $u+v \in \mathcal{C}$; 
(ii) se $u \in \mathcal{C}$ então $\lambda u \in \mathcal{C}$ para todo $\lambda \in \mathbb{R}^{+}$.

(iii) se $u,-u \in \mathcal{C}$ então $u=0$;

Em outras palavras um cone convexo é um conjunto fechado por combinações lineares com coeficientes não negativos que não contém nenhum subespaço vetorial.

Salientamos que as definições de cone podem variar de área para área. A definição acima pode ser em alguns textos o que se chama de cone com ponta (pointed cone) como em Drăgan et al. (2009) ou cone próprio como Schaefer e Wolff (1999).

Definição 5. Um cone convexo $\mathcal{C}$ em um espaço de Hilbert $V$ com produto interno $\langle\cdot, \cdot\rangle$ é dito

- reprodutor se para todo $u \in V$ existem $v, w \in \mathcal{C}$ tal que $u=v-w$.

- autodual se $\langle u, v\rangle \geq 0$ para todo $v \in \mathcal{C}$ se e somente se $u \in \mathcal{C}$

Observação 1) Uma ordem parcial em um conjunto $A$ é uma relação binária $\preceq$ que satisfaz

(i) $a \preceq a$ (propriedade reflexiva).

(ii) se $a \preceq b$ e $b \preceq a$ então $a=b$ (propriedade antissimétrica).

(iii) se $a \preceq b$ e $b \preceq c$ então $a \preceq c$ (propriedade transitiva).

Um cone convexo $\mathcal{C}$ induz uma ordem parcial no espaço vetorial da seguinte forma $u \preceq v$ se e somente se $v-u \in \mathcal{C}$.

Definição 6. Dizemos que uma norma $(\|\cdot\|)$ é monótona com respeito a um cone se $0 \preceq x \preceq y$ então $\|x\| \leq\|y\|$

A próxima proposição é um caso particular da proposição 2.4 do livro de Drăgan et al. (2009).

Proposição 3. Seja $\mathcal{C}$ um cone em um espaço vetorial com produto interno. Se o cone é auto dual então a norma naturalmente derivada do produto interno é monótona.

Demonstração. Seja $0 \preceq x \preceq y$, como o cone é autodual, $\langle y+x, y-x\rangle \geq 0$, $\operatorname{logo}$ $\|x\| \leq\|y\|$.

Definição 7. Um operador $T: V \rightarrow V$ é dito positivo com respeito ao cone convexo $\mathcal{C}$, se a imagem do cone pelo operador $T$ está contida no cone, isto é, $T(\mathcal{C}) \subset \mathcal{C}$.

O próximo teorema é de fundamental importância neste trabalho e é um resultado clássico na área de cones. Iremos apresentar uma versão mais forte que a apresentada usualmente. A prova dada é a de (Deimling, 1985, Teorema 19.2).

Teorema 2 (Teorema de Krein-Rutman). Seja $\mathcal{C}$ um cone convexo fechado reprodutivo autodual em um espaço vetorial com produto interno de dimensão finita $V$. Se Té um operador linear positivo, então $\rho(T)$ é um autovalor e existe um $\bar{X} \in \mathcal{C}$ não nulo tal que $T(\bar{X})=\rho(T) \bar{X}$. 
Demonstração. Seja $\lambda_{0}$ um autovalor associado ao raio espectral $\rho(T)$ e seja $R(\lambda)$ o resolvente de $T$, isto é

$$
R(\lambda)=(T-\lambda I)^{-1}
$$

Os autovalores de um operador linear em espaços de dimensão finita são singularidades isoladas do tipo polo do resolvente, pois para cada autovalor $\lambda, T-\lambda I$ tem ascent Abramovich e Aliprantis, 2002, Definição 2.20) finito e o teorema 6.39 de Abramovich e Aliprantis (2002) implica neste resultado. Logo existe $n$ tal que

$$
\lim _{\lambda \rightarrow \lambda_{0}}\left(\lambda-\lambda_{0}\right)^{n} R(\lambda)=S \neq 0
$$

Além disso

$$
T S=S T=\lambda_{0} S
$$

pois

$$
(T-\lambda I) R(\lambda)=R(\lambda)(T-\lambda I)=I,
$$

e multiplicando por $\left(\lambda-\lambda_{0}\right)^{n}$ e tomando o limite quando $\lambda$ tende a $\lambda_{0}$ obtemos o resultado (2.6). Agora, existe $u \in \mathcal{C}$ tal que $S(u) \neq 0$, pois o cone é reprodutivo e $S \neq 0$. Com efeito seja $w \in V$ tal que $S(w) \neq 0$, então existem $u, v \in \mathcal{C}$ tal que $S(w)=S(u)-S(v)$ e um deles não pode ser nulo. Utilizando um argumento análogo vemos que deve existir um $v \in \mathcal{C}$ tal que $\langle v, S(u)\rangle \neq 0$. Considere a função

$$
\phi(\mu)=\left\langle v, R\left(\frac{1}{\mu}\right)(u)\right\rangle=\sum_{k=0}^{\infty}\left\langle v, T^{k}(u)\right\rangle \mu^{k+1},
$$

que é válida para $|\mu|<\left|\lambda_{0}\right|^{-1}$ se $\rho(T)>0$. Observe que devemos ter $\left\langle v, T^{k}(u)\right\rangle>0$ para um número cofinito de termos, caso contrário

$$
0 \neq\langle v, S(u)\rangle=\lim _{\lambda \rightarrow \lambda_{0}}\left(\lambda-\lambda_{0}\right)^{n}\langle v, R(\lambda)(u)\rangle=\lim _{\lambda \rightarrow \lambda_{0}}\left(\lambda-\lambda_{0}\right)^{n} \sum_{k=0}^{m}\left\langle v, T^{k}(u)\right\rangle \lambda^{-k-1}=0
$$

com $|\lambda|>\left|\lambda_{0}\right|=\rho(T)$. Como a série que define $\phi(\mu)$ tem raio de convergência finito, os coeficientes são não negativos e positivos para infinitos valores, a proposição 11 diz que se $r h o(T)>0$, então $\rho(T)^{-1}$ é uma singularidade de $\phi(\mu)$ e portanto $\rho(T)$ é uma singularidade de $R(\lambda)$. É claro que se $\rho(T)=0$ já é singularidade. Resta mostrar que existe um autovetor no cone. Defina $\bar{S}=\lim _{\lambda \rightarrow \rho(T)}(\lambda-\rho(T))^{m} R(\lambda)$. Se escolhermos fazer o limite $\operatorname{com} \lambda>\rho(T)$ pela série

$$
R(\lambda) w=\sum_{k=0}^{\infty} T^{k}(w) \lambda^{-k-1}
$$

obtemos que $\bar{S}$ é positivo. Como $T \bar{S}=\rho(T) \bar{S}$ o resultado segue com $\bar{X}=\bar{S} w$, com $w \in \mathcal{C}$.

Para finalizarmos esta subseção vamos mostrar que $S_{n}^{+}$é um cone e $\mathcal{T}$ um operador positivo que satisfazem as hipóteses do teorema de Krein-Rutman. A prova da auto dualidade é baseada em (Boyd e Vandenberghe, 2004, Exemplo 2.24). 
Proposição 4. $S_{n}^{+N}$ é um cone convexo, reprodutor, fechado e auto dual em $S_{n}^{N}$. $\mathcal{T}$ é um operador positivo com respeito ao cone.

Demonstração. Para começar, mostraremos que basta provar o resultado para as matrizes simétricas positivas semidefinidas. Seja $\mathcal{C}$ um cone convexo, fechado, sólido e auto dual em um espaço de Hilbert $V$ com produto interno $(\cdot, \cdot)$. Considere o espaço de Hilbert produto de $n$ cópias de $V, V^{n}$, com produto interno $\langle u, v\rangle=\sum_{i=1}^{n}\left(u_{i}, v_{i}\right)$, onde $u_{i}$ a i-ésima coordenada de $u$. $\mathcal{C}^{n}$ é um cone convexo pois $u, v \in \mathcal{C}^{n}$ então $u+v=\left(u_{1}+v_{1}, \ldots, u_{n}+v_{n}\right)$, como $u_{i}+v_{i} \in \mathcal{C}$ para todo $i$ então $u+v \in \mathcal{C}^{n}$, da mesma forma para o produto por escalar por não negativo e se $u,-u \in \mathcal{C}^{n}$ então $u_{i},-u_{i} \in \mathcal{C} \operatorname{logo} u_{i}=0$ para todo $i$. Seja $u \in V^{n}$, então $u_{i} \in V$ para cada $i$ e como $\mathcal{C}$ é reprodutor exitem $v_{i}, w_{i} \in \mathcal{C}$ tal que $u_{i}=v_{i}-w_{i}$ assim definido $v, w$ com coordenadas $v_{i}, w_{i}$ respectivamente temos que $u, v \in \mathcal{C}^{n}$ e $u=v-w$, logo é o cone produto é reprodutor. Seja $\pi_{i}$ a i-ésima projeção de $V^{n}$ em $V$, segue que $\mathcal{C}^{n}=\pi_{1}^{-1}(\mathcal{C}) \cap \cdots \cap \pi_{n}^{-1}(\mathcal{C})$. Como cada projeção é contínua, $\mathcal{C}^{n}$ é fechado. Considere o produto interno entre um elemento de $V^{n}, u$, e elementos de $\mathcal{C}^{n}, v^{i}$, $\operatorname{com} v^{i}=(0, \ldots, \underbrace{w}_{i}, \ldots, 0)$ com $w \in \mathcal{C}$. Segue que $\left\langle u, v^{i}\right\rangle \geq 0$ implica que $\left(u_{i}, w\right) \geq 0$, como $\mathcal{C}$ é autodual e $w$ arbitrário em $\mathcal{C}$, temos $u_{i} \in \mathcal{C}$ para cada $i$, logo $u \in \mathcal{C}^{n}$. Se $u, v \in \mathcal{C}^{n}$ então $(u, v)=\sum_{i=1}^{n}\left\langle u_{i}, v_{i}\right\rangle \geq 0$ o que mostra que o cone produto é autodual.

Chamamos de $S_{n}$ o conjunto das matrizes simétricas de ordem $n$ e $S_{n}^{+}$o subconjunto das matrizes positivas semidefinidas. Estaremos supondo que o produto interno definido em $S_{n}$ é dado por $\langle A, B\rangle=\operatorname{tr}(A B)$. Se $A \in S_{n}^{+}$e $B \in S_{n}$ então $A+B \in S_{n}^{+}$uma vez que $x^{\prime}(A+B) x=x^{\prime} A x+x^{\prime} B x \geq 0$ para todo $x \in \mathbb{R}^{n}$. O mesmo pode ser dito de $\lambda A$ com $\lambda \geq 0$. Se $A,-A \in S_{n}^{+}$então $x^{\prime} A x \geq 0$ e $-x^{\prime} A x \geq 0$, logo $x^{\prime} A x=0$ para qualquer $x$. Como $A$ é simétrica temos que $x^{\prime} A y=\left[(x+y)^{\prime} A(x+y)-x^{\prime} A x-y^{\prime} A y\right] / 2$, portanto $x^{\prime} A y=0$ para todo $x$ e $y$, tomando $x=A y$, vem que $\|A y\|=0$ e assim $A y=0$ para todo $y$, ou seja, $A=0$, o que mostra que $S_{n}^{+}$é um cone convexo. Seja $A \in S_{n}^{+}$, temos que para qualquer matriz real $M M^{\prime} A M$ é também uma matriz simétrica positiva semidefinida pois $x^{\prime} M^{\prime} A M x=(M x)^{\prime} A(M x) \geq 0$. O teorema espectral para matrizes simétricas reais, teorema 8 no apêndice, afirma que se $A$ é uma matriz simétrica existe $U$ tal que $U^{-1}=U^{\prime}$ e $D=U^{-1} S U$ é uma matriz diagonal real. Vamos denotar por

$$
D=\left[\begin{array}{cccc}
\lambda_{1} & 0 & \ldots & 0 \\
0 & \lambda_{2} & \ldots & 0 \\
\vdots & \vdots & \ddots & \vdots \\
0 & 0 & \ldots & \lambda_{n}
\end{array}\right]
$$

a matriz $S$ diagonalizada. Representaremos a matriz $D$ como a soma de duas matrizes, uma diagonal que contém os autovalores não negativos nas mesmas posições e 0 zero nas demais posições que chamaremos de $D^{+}$e uma contenha os negativos nas mesmas posições e zero nas demais que chamaremos de $D^{-}$. É claro que $D^{+},-D^{-}$são positivas semidefinidas e que $D=D^{+}-\left(-D^{-}\right)$. Multiplicando a direita por $U$ e a esquerda por $U^{-1}$ ambos os dois membros da equação obtemos

$$
A=U^{-1} D^{+} U-U^{-1}\left(-D^{-}\right) U=U^{\prime} D^{+} U-U^{\prime}\left(-D^{-}\right) U
$$


onde $U^{\prime} D^{+} U, U^{\prime}\left(-D^{-}\right) U$ são positivas semidefinidas, assim $S_{n}^{+}$é reprodutor. Seja $A \in S_{n}$, mas com $A \notin S_{n}^{+}$, então existe $x \in \mathbb{R}^{n}$ tal que $x^{\prime} A x<0$. Para este $X$ definimos a função

$$
\begin{aligned}
f_{x}: S_{n}^{+} & \rightarrow \mathbb{R} \\
B & \mapsto x^{\prime} B x
\end{aligned}
$$

que é contínua, logo, existe $\delta$ tal $\|A-B\|<\delta$ implica que $x^{\prime} B x<0$. Sendo assim toda matriz que não pertence ao cone é um ponto interior, ou seja, o complementar do cone é aberto, logo o cone é fechado. Vamos verificar que o cone é autodual, mostremos primeiro que se $A \notin S_{n}^{+}$então existe $B \in S_{n}^{+}$tal que $\operatorname{tr}(A B)<0$. De fato, existe $x$ tal que $x^{\prime} A x<0$, utilizando as propriedades do traço $x^{\prime} A x=\operatorname{tr}\left(x x^{\prime} A\right)<0$ e $x x^{\prime}$ é positiva semidefinida pois $y^{\prime} x x^{\prime} y=\left(x^{\prime} y\right)^{2} \geq 0$. Isto mostra que se $\langle A, B\rangle \geq 0$ para todo $b \in S_{n}^{+}$então $a \in S_{n}^{+}$. Se $A, B \in S_{n}^{+}$, então existe $B^{\frac{1}{2}} \in S_{n}^{+}$. Segue que $\operatorname{tr}(A B)=\operatorname{tr}\left(A B^{\frac{1}{2}} B^{\frac{1}{2}}\right)=\operatorname{tr}\left(B^{\frac{1}{2}} A B^{\frac{1}{2}}\right)$, mas $B^{\frac{1}{2}} A B^{\frac{1}{2}} \in S_{n}^{+}$, pois $A \in S_{n}^{+}$e $\left(B^{\frac{1}{2}}\right)^{\prime}=B^{\frac{1}{2}}, \operatorname{logo} \operatorname{tr}(A B) \geq 0$ lembrando que o traço de $A B$ é a soma dos autovalores de $B^{\frac{1}{2}} A B^{\frac{1}{2}}$ que são não negativos. Logo $S_{n}^{+}$é um cone convexo, reprodutor, fechado, auto dual. Como $S_{n}^{+N}=\left(S_{n}^{+}\right)^{N}$ o resultado segue pelo começo da demonstração.

Resta verificar que $\mathcal{T}$ é um operador positivo. Se $X \in S_{n}^{+N}$ então $\mathcal{T}_{i}(X)=$ $\sum_{j=1}^{N} p_{j i} A_{j} X_{j} A_{j}^{\prime} \in S_{n}^{+}$pois cada termos é combinação linear com coeficiente não negativos de termos positivos semidefinidos, $\log \mathrm{T}(X) \in S_{n}^{+N}$, o que conclui a demonstração.

Denotaremos a ordem parcial em $S_{n}^{N}$ induzida pelo cone $S_{n}^{+N}$ por $\preceq$ e a ordem oposta por $\succeq$,isto é, $A \preceq B$ se e somente se $B \succeq A$.

\subsubsection{Custo médio a longo prazo e Detetabilidade}

Nesta subseção vamos definir o custo médio a longo prazo e a detetabilidade fraca.

Definição 8. O custo de horizonte t com condição inicial $x(0), \theta(0)$ associado ao sistema $(A, G, Q, P)$ é definido por

$$
J(t, x(0), \theta(0))=\sum_{k=0}^{t} E[c(k)] .
$$

Proposição 5. Para o sistema $(A, G, Q, P)$ temos que

$$
J(t, x(0), \theta(0))=\sum_{k=0}^{t}\langle Q, X(k, X)\rangle
$$

onde $X=X(0)$ como em 2.5$)$.

Demonstração.

$$
E(c(k))=E\left(x(k)^{\prime} Q_{\theta(k)} x(k)\right)=E\left(\operatorname{tr}\left(x(k)^{\prime} Q_{\theta(k)} x(k)\right)\right),
$$

pois $x(k)(\omega)^{\prime} Q_{\theta(k)(\omega)} x(k)(\omega)$ é um número real. Como tr é um operador linear e portanto comuta com a média e $\operatorname{tr}(A B)=\operatorname{tr}(B A)$ segue que

$$
E(c(k))=\operatorname{tr}\left(E\left(x(k) x(k)^{\prime} Q_{\theta(k)}\right)\right)=\operatorname{tr}\left(E\left(\sum_{i=0}^{N} x(k) x(k)^{\prime} Q_{i} 1_{\{\theta(k)=i\}}\right)\right),
$$


onde foi usado que $\sum_{i=1}^{N} 1_{\{\theta(k)=i\}}=1$ para qualquer $f$ mensurável e que $f(\theta(k)) 1_{\{\theta(k)=i\}}=$ $f(i) 1_{\{\theta(k)=i\}}$.

$$
E(c(k))=\sum_{i=1}^{N} \operatorname{tr}\left(E\left(x(k)^{\prime} x(k) 1_{\{\theta(k)=i\}}\right) Q_{i}\right)=\sum_{i=1}^{N} \operatorname{tr}\left(X_{i}(k, X) Q_{i}\right)=\langle Q, X(k)\rangle .
$$

Enfim segue que

$$
J(t, x(0), \theta(0))=\sum_{i=0}^{t}\langle Q, X(k, X)\rangle=: J(t, X) .
$$

Devido a esta proposição passaremos a utilizar apenas a notação $J(t, X):=$ $J(t, x(0), \theta(0))$. A definição do custo médio a longo prazo a seguir é comum na área de sistemas lineares com saltos markovianos, veja por exemplo Costa et al., 2005, Capítulo 4) ou Vargas et al. (2006) para uma abordagem mais próxima da nossa.

Definição 9 (Custo médio a longo prazo). O custo médio a longo prazo associado ao sistema $(A, G, Q, P)$ com condição inicial $X$ é definido como

$$
\mathcal{J}(X)=\limsup _{t} \frac{J(t, X)}{t} .
$$

No caso em que o custo médio a longo prazo existe para todo $X \in S_{n}^{+N}$, dizemos que o custo médio a longo prazo é limitado se existe $\bar{J}$ tal que $\mathcal{J}(X)<\bar{J}$ qualquer que seja $X$.

Proposição 6. O custo médio a longo prazo associado ao sistema sistema $(A, G, Q, P)$ é limitado se e somente se existe $\bar{J}$ de forma que para todo $X \in S_{n}^{+N}$ existe $t_{0}$ tal que

$$
J(t, X)<\bar{J} t
$$

para todo $t>t_{0}$.

Demonstração. Dada uma sequência $\left(x_{t}\right)_{t \in \mathbb{N}}$ em $\mathbb{R}$ se existem $M, N, t_{0}$ tais que

$$
A \leq x_{t}<B
$$

para $t>t_{0}$ então o $\lim \sup x_{t}$ existe e satisfaz

$$
A \leq \lim \sup \left(x_{t}\right)<B
$$

se vale a equação (2.7) temos que o custo médio a longo prazo existe e satisfaz

$$
0 \leq \mathcal{J}(X)<\bar{J}
$$

Reciprocamente se o custo médio a longo prazo satisfaz 2.8) então para

$$
0 \leq \frac{J(t, X)}{t} \leq \mathcal{J}(X)+\epsilon<\bar{J}
$$

para $t_{0}$ dependendo de $X$ suficientemente grande. 
A definição de detetabilidade fraca que segue foi dada em (Costa e do Val, 2001, Definição 5), sendo inspirada na definição de (Kalman, 1960, Definição 5.23) e (Anderson e Moore, 1981, Definição 2.1).

Definição 10 (Detetabilidade Fraca). Dizemos que o sistema $(A, 0, Q, P)$ é fracamente detetável se existem $t_{d}>0, s_{d}>0, \gamma>0$ e $\delta \in[0,1)$ tais que para todo $X \in S_{n}^{+N}$ vale que

$$
J\left(t_{d}-1, X\right) \geq \gamma\|X\| \quad \text { quando } \quad\left\|X\left(s_{d}, X\right)\right\| \geq \delta\|X\| .
$$

Se a definição anterior vale com $\delta=0$ dizemos que o sistema é fracamente observável. O próximo teorema foi obtido em (Costa e do Val, 2001, Lema 8). Fornecemos uma prova diferente, pois esta prova é construtiva.

Teorema 3. O sistema $(A, 0, Q, P)$ é fracamente detetável se e somente se para todo $X \in S_{n}^{+N}$ tal que $\left\langle Q, \mathcal{T}^{t}(X)\right\rangle=0$ para todo $t$ vale que $\lim _{t \rightarrow \infty} \mathcal{T}^{t}(X)=0$.

Demonstração. $(\Rightarrow)$ Suponha que $(A, 0, Q, P)$ é detetável, então, quando $\left\langle Q, \mathcal{T}^{t}(X)\right\rangle=0$ para todo $t$, temos pela condição de detetabilidade

$$
\left\|\mathcal{T}^{i+s n}(X)\right\|<\delta^{n}\left\|\mathcal{T}^{i}(X)\right\| .
$$

Segue que

$$
\left\|\mathcal{T}^{n}(X)\right\|<\delta\left\lfloor\frac{n}{s}\right\rfloor \max _{0 \leq i<s}\left\|\mathcal{T}^{i}(X)\right\|
$$

$(\Leftarrow)$ Seja $E \subset S_{N}^{n}$ o subconjuntos $\operatorname{dos}_{\lim _{i \rightarrow \infty}} \mathcal{T}^{i}(X)=0$. Pelo teorema 1 (mais precisamente pela demonstração), o operador $\mathcal{T}$ restrito $E$ é exponencialmente estável, isto é, existem $\beta$ e $\xi$ tais que $\left\|\mathcal{T}^{i}(X)\right\|<\alpha \xi^{i}\|X\|$ para todo $X \in E$. Seja $s_{d}$ o menor inteiro tal que $\delta:=\beta \xi^{s_{d}}<1$ Defina o conjunto $C=\left\{X \in S_{n}^{N}:\left\|\mathcal{T}^{s_{d}}\right\|<\delta\|X\|\right\}$. Temos que este conjunto é aberto, pois $\left\|\mathcal{T}^{s_{d}}(X)\right\|-\delta\|X\|$ é contínua, claramente $E \subset C$. Considere $F$ o menor subespaço que contenha $X \in S^{+}$para os quais $\left\langle Q, \mathcal{T}^{i}(X)\right\rangle=0$. Para todo $X \in F,\left\langle Q, \mathcal{T}^{i}(X)\right\rangle=0$. Além disso vale $F \subset E$. Denotemos por $C^{c}$ e $F^{c}$ os complementares de $Q$ e $F$, segue que $C^{c} \subset F^{c}$, logo para todo $X \in C^{c}$ existe $i$ tal que $\left\langle Q, \mathcal{T}^{i}(X)\right\rangle \neq 0$. Devemos ter $\left\langle Q, \mathcal{T}^{i}(X)\right\rangle \neq 0$, para $i<(n+1) n / 2$ pois pelo teorema de Cayley-Hamilton 9 , potências iguais ou maiores do que a dimensão do espaço $S_{n}^{N}$ se escrevem como combinação linear de potências menores. Seja $t_{d}=n(n+1) / 2$, temos que para todo $X \in C^{c}$

$$
\sum_{i=0}^{t_{d}}\left\langle Q, \mathcal{T}^{i}(X)\right\rangle \neq 0 .
$$

Observe que para $\alpha \neq 0$ implica $\alpha X \in C^{c}$. Seja $S$ o círculo unitário, isto é, $S=\{X \in$ $\left.S_{n}^{N}:\|X\|=1\right\}$. O círculo unitário é compacto pois estamos tratando de um espaço de dimensão finita. Considere a interseção $C^{c} \cap S \cap S_{n}^{+N}$. Este conjunto é compacto, pois todos os conjuntos são fechados e $S$ é compacto. Vale que para todo $X \in C^{c} \cap S_{n}^{+N}$.

$$
\sum_{i=0}^{t_{d}}\left\langle Q, \mathcal{T}^{i}(X)\right\rangle>\gamma\|X\| .
$$


Ou seja, temos que $S_{n}^{+N}=\left(C \cap S_{n}^{+N}\right) \cup\left(C^{c} \cap S_{n}^{+N}\right)$. O que mostra que o sistema é detetável.

O próximo resultado desta seção foi provado inicialmente em (Vargas et al., 2006, Corolário 1) para cadeias de Markov ergódicas e estendido em (Costa et al., 2011, Teorema 2) para o caso geral. Nos artigos citados os objetivos vão além do próximo teorema e por conseguinte a prova é extensa. Aqui forneceremos uma prova mais direta.

Teorema 4. Se o raio espectral de $\mathcal{T}$ é menor que um, então o custo médio a longo prazo associado ao sistema $(A, G, Q, \mathbf{P})$ é limitado.

Demonstração. Lembramos que pelo teorema 11 sistema é exponencialmente estável, isto é,

$$
\left\|\mathcal{T}^{t}(X)\right\| \leq \beta \xi^{t}\|X\|
$$

Considere a evolução dada por (2.5), temos fazendo o produto interno com $Q$

$$
\langle Q, X(t)\rangle=\left\langle Q, \mathcal{T}^{t}(X)\right\rangle+\left\langle Q, \sum_{i=0}^{t-1} \mathcal{T}^{i}\left(\Sigma_{t-1-i}\right)\right\rangle
$$

e utilizando a desigualdade de Cauchy-Swarchz e a estabilidade exponencial

$$
\langle Q, X(t)\rangle \leq\|Q\| \beta \xi^{t}\|X\|+\|Q\| \sum_{i=0}^{t-1} \beta \xi^{i}\left\|\Sigma_{t-1-i}\right\| .
$$

Defina $M:=\sum_{i=1}^{N}\left\|G_{i} G_{i}^{\prime}\right\|$ e observe que $\left\|\Sigma_{i}\right\| \leq\|M\|$, então

$$
\langle Q, X(t)\rangle \leq\|Q\| \beta\left(\xi^{t}+\|M\| \sum_{i=0}^{t-1} \xi^{i}\right)
$$

e utilizando que $\sum_{i=0}^{t-1} \xi^{i} \leq 1 /(1-\xi)$ obtemos

$$
\langle Q, X(t)\rangle \leq\|Q\| \beta\left(\xi^{t}\|X\|+\|M\| \frac{1}{1-\xi}\right) .
$$

Somamos em $t$ para obter o custo

$$
J(t, X) \leq\|Q\| \beta \frac{1}{1-\xi}(\|X\|+t\|M\|)
$$

e assim temos que

$$
\frac{J(t, X)}{t} \leq\|Q\| \beta \frac{1}{1-\xi}\|M\|+1
$$

para $t$ suficientemente grande dependendo de $X$, segue pelos argumentos da proposição 6 que o custo médio a longo prazo existe e é limitado.

A proposição final da seção é o lema 12 de Costa e do Val (2001). Apresentamos a prova por facilidade de referência. 
Proposição 7. Se o sistema $(A, 0, Q, \mathbf{P})$ é fracamente detetável então o sistema $(A+$ $\left.B L, 0, Q+L^{\prime} R L, \mathbf{P}\right)$ é fracamente detetável $L \in M_{s, n}^{N}$ e $R$ constituído de matrizes positivas definidas.

Demonstração. Vamos denotar a evolução das matrizes de segundo momento condicionada do sistema $\left(A+B L, 0, Q+L^{\prime} R L, \mathbf{P}\right)$ por $X(t, X)$. Observe que $\left\langle Q+L^{\prime} R L, X(t, X)\right\rangle=$ 0 se e somente se $\langle Q, X(t, X)\rangle=0$ e $\left\langle L^{\prime} R L, X(t, X)\right\rangle=0$. Desenvolvendo a última expressão temos que $\left\langle R^{\frac{1}{2}} L X(t, X)^{\frac{1}{2}}, R^{\frac{1}{2}} L X(t, X)^{\frac{1}{2}}\right\rangle=\left\|R^{\frac{1}{2}} L X(t, X)^{\frac{1}{2}}\right\|=0$, o que implica que $L X(t, X)=0$ pois $R$ é positiva definida. Temos em relação a evolução que,

$$
X_{i}(t+1, X)=\sum_{j=1}^{N} p_{j i}\left(A_{j}+B_{j} L_{j}\right) X_{j}(t, X)\left(A_{j}+B_{j} L_{j}\right)^{\prime}=\sum_{j=1}^{N} p_{j i} A_{j} X_{j}(t, X) A_{j}^{\prime},
$$

assim, como $\langle Q, X(t, X)\rangle=0$ e $(A, 0, Q, \mathbf{P})$ é detetável, pela expressão anterior $\lim _{t \rightarrow \infty} X(t, X)=0$ o que mostra que $\left(A+B L, 0, Q+L^{\prime} R L, \mathbf{P}\right)$ é detetável.

Finalmente estamos aptos a formular o problema de uma forma mais precisa. O objetivo de investigação será o seguinte:

- É verdade que custo médio a longo prazo limitado e detetabilidade fraca são condições necessárias e suficientes para atingir estabilidade assintótica no sentido de Lagrange?

Responderemos afirmativamente esta pergunta na seção 3.1 do próximo capítulo.

\subsection{Sistemas dinâmicos generalizados}

Nesta seção vamos introduzir as definições e hipóteses necessárias para tratarmos sistemas dinâmicos generalizados. É importante notar que existem várias formas de generalização para sistemas dinâmicos, desde da abordagem comportamental de Willems (1991), passando pelo general system de Zubov (1964) e chegando aos motions de Hahn e Baartz (1967) que é a definição mais próxima de que utilizaremos aqui. Mais precisamente utilizamos uma variante da definição de Michel et al. (2001, 2008) onde vamos considerar o instante inicial a partir do qual se observa a trajetória no espaço de estados como sendo o instante zero. Esta restrição por sua vez não necessariamente limita o trabalho a sistemas autônomos, porém as hipóteses que serão feitas sobre os sistemas dinâmicos generalizados que estaremos analisando certamente aproximam estes de sistemas autônomos e os contém como subclasse.

Definição 11. Seja $M$ um espaço métrico, que chamaremos de espaço de estados, e $A \subset M$, chamado de condições iniciais. Considere uma coleção de subconjuntos de $T_{a}=$ $\left\{t \in \mathbb{N}: t \leq t_{1}\right\}$, para algum $t_{1} \in \mathbb{N}$ ou $t_{1}=\infty$, para cada $a \in A$ e uma coleção de funções $x(\cdot, a): T_{a} \rightarrow M$ tais que $x(0, a)=a$. Cada $x$ é chamado de moviment 1 e a coleção de movimentos sobre $(M, A)$ será denotada por $S$.

\footnotetext{
${ }^{1}$ Tradução imediata do inglês motion
} 
Definição 12. Seja $M$ um espaço métrico, $A \subset M$, e $S$ uma família de movimentos sobre $(M, A)$. Dizemos que a túpla $(M, \mathbb{N}, A, S)$ é um sistema dinâmico generalizado a tempo discreto com condições iniciais em A. Em geral identificamos o sistema dinâmico com a família de movimentos.

Antes de prosseguir faremos a observação que esta definição foi feita com o tempo sendo o conjunto dos números naturais para se adequar melhor as nossas necessidade, mas nada implica que não se possa utilizar outro conjunto totalmente ordenado qualquer, o que é feito por exemplo em casos de sistemas híbridos.

Suponha que a cada $p \in M$ associamos um custo não negativo $c: M \rightarrow \mathbb{R}^{+}$. Desta forma é natural associar a cada movimento o custo de execução do movimento até um instante $n$ definido como a soma dos custos em cada estado ocupado até o instante de interesse, isto é

$$
\begin{aligned}
W: \mathbb{N} \times S \times A & \rightarrow \mathbb{R}^{+}, \\
W(n, x, a) & =\sum_{i=0}^{n} c(x(i, a)) .
\end{aligned}
$$

A forma deste custo é importante para este trabalho, pois iremos utilizar sua aditividade mais adiante, mais precisamente no lema 2. Dizemos que um sistema dinâmico tem um ponto estacionário, fixo ou de equilíbrio $e \in M$ se para to $x \in S$ temos que $x(n, e)=e$ para todo $n \in \mathbb{N}$. A partir do custo podemos definir a detetabilidade fraca com relação a um ponto estacionário $e$

Definição 13 (Detetabilidade Fraca). Dizemos que o sistema dinâmico generalizado $(M, \mathbb{N}, A, S)$ é fracamente detetável em relação a um ponto estacionário e se existem $t>0, s>0 \gamma>0$ e $\delta \in[0,1) x \in S$ e $a \in A$

$$
W(t-1, x, a) \geq \gamma d(a, e) \text { sempre que } d(x(s, a), e) \geq \delta d(a, e) .
$$

Esta definição encontra-se fundamentada para sistemas lineares onde o custo é o gramiano de observabilidade. Para a maioria dos sistemas dinâmicos nos quais a observação de um estado se dá através de uma função do estado $h: M \rightarrow N$ onde $N$ é um outro espaço métrico com métrica $\hat{d}$ é natural esperar que o custo de observação seja da forma $c(a)=\hat{d}(h(a), h(e))$, ou seja o custo é pago a medida que o estado observado difere de outro estado, quanto maior custo pago maior o nível de observação. É importante salientar que desconhecemos testes de detetabilidade fraca para além do caso linear.

O que vamos mostrar para sistemas dinâmicos generalizados é que em uma situação em que o custo médio a longo prazo é limitado a detetabilidade do sistema implica em estabilidade no sentido de Lagrange. Vamos escrever esta definição de estabilidade para o caso dos sistemas de interesse

Definição 14 (Estabilidade assintótica no sentido de Lagrange). Dizemos que o sistema $(M, \mathbb{N}, A, S)$ é assintoticamente estável no sentido de Lagrange em relação ao ponto de equilíbrio e se existe $r$ e para cada $x \in S$ e a $\in A$ existe $n_{0}$ tal que para todo $n>n_{0}$ temos

$$
d(x(n, a), e)<r
$$


É necessário fazer algumas hipóteses sobre a família de movimentos $S$ que vamos tratar. Estaremos interessados numa família de movimentos que satisfaz as seguintes hipóteses:

H1) Todo ponto do espaço $M$ é condição inicial, ou seja, $A=M$.

H2) Existe um ponto estacionário $e$.

H3) Se $x$ é um movimento, então existe um movimento $y$ tal que $y(\cdot, x(n, a))=x(n+\cdot, a)$ para todo $a \in A$. Isto quer dizer que toda continuação de um movimento é um movimento.

H4) Para todo movimento $x \in S$ e para todo $b \in A, d(x(n+1, a), e) \leq \operatorname{Ld}(x(n, a), e)$, para algum $L$.

A respeito do custo fazemos a hipótese:

H1') Existe $\bar{W}$ e uma função $g: \mathbb{R}^{+} \rightarrow \mathbb{R}^{+}$não decrescente tal que $W(n, x, a) \leq \bar{W} n+$ $g(d(a, e))$

Esta última hipótese implica que o custo médio a longo prazo existe para todo movimento e condição inicial, com efeito, o custo médio a longo prazo é definido como

$$
\mathcal{W}(x, a)=\limsup _{n} \frac{W(n, x, a)}{n},
$$

que existe quando $W(n, x, a) / n$ é limitado assintoticamente superior e inferiormente, mas temos que neste caso qualquer que seja $x, a$, este termo é limitado inferiormente por $0 \mathrm{e}$ superiormente por $\bar{W}+1$ para $n$ grande. Neste ponto ocorre uma diferença em relação ao caso de sistemas lineares com saltos markovianos: aqui a condição sobre o custo apenas implica em sua limitação, não sendo equivalente a mesma.

Para mostrar que estas restrições aos movimentos não produz um conjunto vazio de movimentos, vamos verificar que de fato elas são satisfeitas pelo sistema dinâmico gerado por uma função $f: M \rightarrow M$. Suponha que todo movimento seja da seguinte forma $x(n, a)=f^{n}(a)$ onde $f$ é Lipchitz e tem um ponto fixo $e$. Temos que todo $x(\cdot, a)$ está definida para todo $a \in M$. O ponto $e$ é um ponto de equilíbrio do movimento pois $x(n, e)=f^{n}(e)=e$ para qualquer $n$. Existe o movimento $y(, x(n, a))=x(n+\cdot, a)$, basta tomar $y=x$, pois $f^{k}\left(f^{n}(a)\right)=f^{k+n}(a)$. Por fim $d(x(n+1, a), e)=d\left(f\left(f^{n}(a)\right), f(e)\right) \geq$ $L d\left(f^{n}(a), f(e)\right)=d(x(n, a), e)$ onde a segunda desigualdade decorre do fato de $f$ ser Lipchitz.

Outros dois exemplos que satisfazem estas hipóteses sobre o movimento são as inclusões a diferenças finitas, isto é, uma função $F: M \rightarrow \mathcal{P}(M)$ onde $\mathcal{P}(M)$ é o conjunto das partes de $M$ tal que exista um $e$ tal que $F(e)=\{e\}$ e satisfaça uma condição próxima a Lipchitz, como por exemplo $d(F(x), F(y)) \subset[0, L d(x, y)]$ e que definimos os movimentos com soluções de

$$
x_{n} \in F\left(x_{n-1}\right),
$$

e a união de sistemas, isto é, dados dois sistemas que satisfazem as hipóteses com mesmo $e$, a união dos conjuntos de movimentos de cada um também satisfaz as hipóteses. 


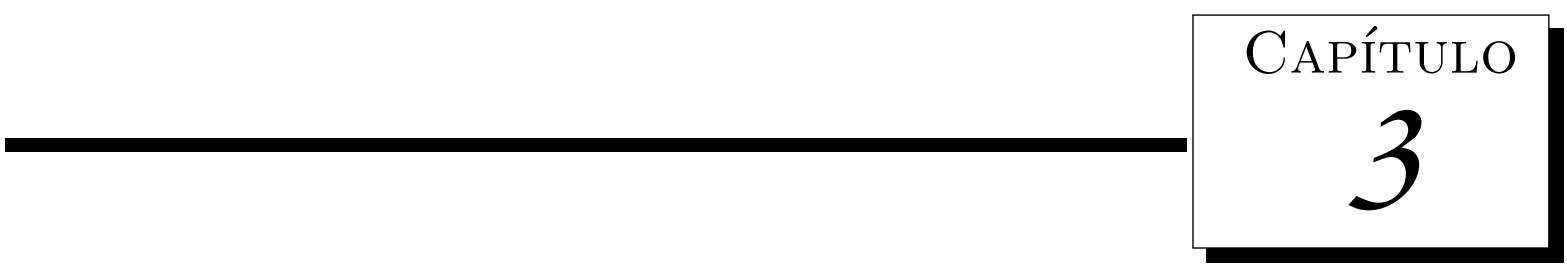

\section{Detetabilidade e custo médio a longo prazo e estabilidade}

Este capítulo contém os resultados mais importantes desenvolvidos durante a pesquisa. Todos os resultados aqui são originais ou desenvolvidos de forma independente de outros autores.

De forma análoga ao capítulo anterior, apresentamos primeiro o resultado para sistema lineares com saltos markovianos e depois o resultado para sistemas dinâmicos generalizados.

\subsection{Sistemas Lineares com Saltos Markovianos}

Os resultados desta seção foram submetidos ao Institute of Electrical and Electronics Engineers Transactions in Automatic Control (IEEE/TAC) Barbosa e Costa (2012a).

\subsubsection{Teoremas principais}

O primeiro teorema que vamos provar aborda a equivalência entre a estabilidade assintótica no sentido de Lagrange de um sistema com a estabilidade em média quadrática do sistema homogêneo associado.

Teorema 5. O sistema $(A, G, Q, \mathbf{P})$ é assintoticamente estável no sentido de Lagrange se e somente se $\rho(\mathcal{T})<1$.

Demonstração. Suficiência $(\Rightarrow)$. Como $\rho(\mathcal{T})<1$ pelo teorema 1 ocorre que o sistema $(A, 0, Q, \mathbf{P})$ é exponencialmente estável na média quadrática, isto é, existem $\beta>0 \mathrm{e}$ $0 \leq \xi<1$ tais que, para todo $t$ e $X$,

$$
\left\|\mathcal{T}^{t}(X)\right\| \leq \beta \xi^{t}\|X\| .
$$


Tomando a norma na equação 2.5

$$
\|X(t, X)\|=\left\|\mathcal{T}^{t}(X)+\sum_{i=0}^{t-1} \mathcal{T}^{i}\left(\Sigma_{t-1-i}\right)\right\|,
$$

e usando a desigualdade triangular chegamos a

$$
\|X(t, X)\| \leq\left\|\mathcal{T}^{t}(X)\right\|+\sum_{i=0}^{t-1}\left\|\mathcal{T}^{i}\left(\Sigma_{t-1-i}\right)\right\| .
$$

Utilizando (3.1) em (3.3), obtemos

$$
\|X(t, X)\| \leq \beta \xi^{t}\|X\|+\sum_{i=0}^{t-1} \beta \xi^{i}\left\|\Sigma_{t-1-i}\right\| .
$$

Agora, defina $M:=\sum_{i=1}^{N}\left\|G_{i} G_{i}^{\prime}\right\|$, e da de (2.3) note que $\left\|\Sigma_{t}\right\| \leq M$ para todo $t$. Isto e (3.4) levam a

$$
\|X(t, X)\| \leq \alpha \xi^{t}\|X\|+\alpha M \sum_{i=0}^{t-1} \xi^{i}
$$

e, levando em consideração que $\sum_{i=0}^{t-1} \xi^{i}<1 /(1-\xi)$, vem que

$$
\|X(t, X)\|=\alpha \xi^{t}\|X\|+\frac{\alpha M}{1-\xi} .
$$

Logo, a definição 3 é satisfeita com $C>(\alpha M) /(1-\xi)$.

Necessidade $(\Leftarrow)$. Vamos provar a contrapositiva, isto é, se $\rho(\mathcal{T}) \geq 1$ então o segundo momento do sistema $(A, G, Q, \mathbf{P})$ não é assintoticamente limitado. Como $\mathcal{T}$ é um operador positivo e $\Sigma_{t} \succeq 0$, o segundo termo a direita da equação (2.5) está em $S_{n}^{+N}$, por isto

$$
X(t, X)-\mathcal{T}^{t}(X) \succeq 0,
$$

e assim $X(t, X) \succeq \mathcal{T}^{t}(X) \succeq 0$ Sendo a norma induzida pelo produto interno em $S_{n}^{N}$ é monótona segue que

$$
\|X(t, X)\| \geq\left\|\mathcal{T}^{t}(X)\right\|
$$

Considere $\bar{X}$ como no teorema 2 e seja $X=\alpha \bar{X} \operatorname{com} \alpha>0$. Aplicando o teorema 2 em (3.5) vem que

$$
\|X(t, \alpha \bar{X})\| \geq\left\|\mathcal{T}^{t}(\alpha \bar{X})\right\|=\rho(\mathcal{T})^{t}\|\alpha \bar{X}\|>\alpha\|\bar{X}\|
$$

e, como podemos escolher $\alpha>C /(\|\bar{X}\|)$ para $C$ na definição 3 , vemos que o sistema $(A, G, Q, P)$ não pode ser assintoticamente estável no sentido de Lagrange.

O resultado acima já foi estudado em outros contextos e até possui generalizações, mas o método de prova é diferente do empregado aqui. Consulte por exemplo (Drăgan et al. 2009, Teorema 2.3).

O próximo resultado é o teorema central da parte deste trabalho que trata de sistemas lineares com saltos markoviano. A ideia básica contida no teorema é que se a dinâmica não paga custo ela é naturalmente estável, e se ela paga então o autovetor associado ao raio espectral paga um custo que viola a limitação do custo médio a longo prazo no caso em que o raio espectral é maior ou igual a 1. Este resultado é original, sendo o primeiro que usa a noção de detetabilidade fraca. 
Teorema 6. O sistema $(A, G, Q, \mathbf{P})$ é assintoticamente estável no sentido de Lagrange se e somente se o sistema $(A, 0, Q, \mathbf{P})$ é fracamente detetável e o custo médio a longo prazo do sistema $(A, G, Q, \mathbf{P})$ é limitado.

Demonstração. Suficiência $(\Rightarrow)$. Seja $\bar{X}$ como no teorema 2 e considere a quantidade

$$
S(t)=\sum_{i=0}^{t}\left\langle Q, \mathcal{T}^{i}(\bar{X})\right\rangle
$$

Vamos dividir o problema em dois casos. $S(t)=0$ para todo $t$ e $S(t) \neq 0$ para algum $t$.

(i) $S(t)=0$ para todo $t$. O sistema $(A, 0, Q, \mathbf{P})$ é detetável então $\lim _{t \rightarrow \infty} \mathcal{T}^{t}(\bar{X})=0$ pelo teorema 3. Logo vem que,

$$
\lim _{t \rightarrow \infty} \rho(\mathcal{T})^{t} \bar{X}=0
$$

que implica $\rho(\mathcal{T})<1$.

(ii) $S(t) \neq 0$ para algum $t$. Neste caso temos $\langle Q, \bar{X}\rangle \sum_{i=0}^{t} \rho(\mathcal{T})^{i} \neq 0$ e por isso $\langle Q, X\rangle \neq$ 0 . Definimos $\beta:=\bar{J} /\langle Q, \bar{X}\rangle$. É verdade que

$$
J(t, X) \geq \sum_{i=0}^{t}\left\langle Q, \mathcal{T}^{i}(X)\right\rangle
$$

pois o termo da equação 2.5 relativo ao ruído, $\sum_{i=0}^{t-1} \mathcal{T}^{i} \Sigma_{t-1-i}$, está no cone $S_{n}^{+N} \mathrm{e}$ $\left\langle Q, \sum_{i=0}^{t-1} \mathcal{T}^{i} \sum_{t-1-i}\right\rangle$ é positivo pela proposição 4. Utilizando a hipótese de existência do custo médio a longo prazo, mais precisamente proposição 6 temos

$$
\bar{J} t>J(t, \beta \bar{X}) \geq \sum_{i=0}^{t}\left\langle Q, \mathcal{T}^{i}(\beta \bar{X})\right\rangle=\beta\langle Q, \bar{X}\rangle \sum_{i=0}^{t} \rho(\mathcal{T})^{i}=\bar{J} \sum_{i=0}^{t} \rho(\mathcal{T})^{i}
$$

o que leva a

$$
t>\sum_{i=0}^{t} \rho(\mathcal{T})^{i}
$$

o que por sua vez implica que $\rho(\mathcal{T})<1$.

Mostramos que $\rho(\mathcal{T})<1$. Em vista do teorema 5 o sistema $(A, G, Q, \mathbf{P})$ é assintoticamente estável no sentido de Lagrange.

Necessidade $(\Leftarrow)$. Invocamos novamente o teorema 5, ou seja, $\rho(\mathcal{T})<1$. Como $\rho(\mathcal{T})<1$ segue pelo teorema 1 que as trajetórias de $(A, 0, Q, \mathbf{P}), X_{0}(t, X)$, satisfazem $\lim _{t \rightarrow \infty} X_{0}(t, X)=0$. Pelo teorema 3 o sistema $(A, 0, Q, \mathbf{P})$ é detetável. Pelo teorema 4 , $\rho(\mathcal{T})<1$ implica que o custo médio a longo prazo é limitado. Isto conclui a demonstração.

Como uma aplicação do teorema anterior a controle de sistemas vamos analisar o seguinte caso. Considere um sistema da forma 


$$
\left\{\begin{array}{l}
x(k+1)=A_{\theta(k)} x(k)+G_{\theta(k)}+B_{\theta} u(k), \\
c(k)=x(k)^{\prime} Q_{\theta(k)} x(k)+u(k)^{\prime} R_{\theta(k)} u(k), \\
y(k)=C_{\theta(k)} x(k),
\end{array}\right.
$$

onde $u(k)=K_{\theta(k)} y(k)$ e $R$ é uma coleção de matrizes positivas definidas. A partir do teorema 6 obtemos o

Corolário 1. Assuma que $(A, 0, Q, \mathbf{P})$ é fracamente detetável. Então o sistema definido em (3.10) com realimentação de saída é assintoticamente estável no sentido de Lagrange se e somente o custo médio a longo prazo associado a este sistema é limitado.

Demonstração. Observe que o sistema (3.10) com realimentação de saída equivale ao sistema $\left(A+B K C, G, Q+C^{\prime} K^{\prime} R K C, \mathbf{P}\right)$. Com o auxílio da proposição 7 vemos que $\left(A+B K C, G, Q+C^{\prime} K^{\prime} R K C, \mathbf{P}\right)$ é fracamente detetável quando $(A, 0, Q, \mathbf{P})$ é fracamente detetável. Logo pelo teorema 6 considerando $\left(A+B K C, G, Q+C^{\prime} K^{\prime} R K C, \mathbf{P}\right)$ o resultado é imediato.

\subsubsection{Exemplos Numéricos}

Considere o sistema (2.1) com $A_{1}=0, A_{2}=\sqrt{2}, G_{1}=1, G_{2}=1$,

$$
Q_{1}=\alpha, \quad Q_{2}=1-\alpha \text { e } \mathbf{P}(\beta)=\left[\begin{array}{cc}
1 & 0 \\
\beta & 1-\beta
\end{array}\right],
$$

onde $\alpha, \beta \in[0,1]$. Temos o seguintes fatos resumidos na tabela 3.1 Estes fatos podem ser Tabela 3.1: Relação entre detetabilidade e estabilidade assintótica no sentido de Lagrange para diferentes valores de $\alpha$ e $\beta$

\begin{tabular}{|c|c|c|c|c|c|}
\cline { 3 - 6 } \multicolumn{2}{c|}{} & \multicolumn{4}{|c|}{$\beta$} \\
\cline { 3 - 6 } \multicolumn{2}{c|}{} & 0 & $(0,1 / 2]$ & $(1 / 2,1)$ & 1 \\
\hline \multirow{4}{*}{$\alpha$} & 0 & I,D & I,D & E,D & E,D \\
\cline { 2 - 6 } & $(0,1)$ & I,O & I,O & E,O & E,O \\
\cline { 2 - 6 } & 1 & I,N & I,O & E,O & E,O \\
\hline
\end{tabular}

\begin{tabular}{|c|c|}
\hline D & Detetável \\
\hline O & Observável \\
\hline N & Não detetável \\
\hline E & Estável \\
\hline I & Instável \\
\hline
\end{tabular}

constatados através dos testes presentes no apêndice, seção A.3.

Vamos analisar o comportamento do custo médio a longo prazo em alguns casos de estudo particulares que retratam bem as particularidades deste sistema apreciado sob a perspectiva do teorema 6 .

Caso 1) ( $\alpha=0$ e $\beta=3 / 4$ ) Pela tabela o sistema é assintoticamente estável no sentido de Lagrange e é fracamente detetável. A partir da equação (3.2) $\lim _{t \rightarrow \infty} X_{1}(t)=1$ e $\lim _{t \rightarrow \infty} X_{2}(t)=0$, resultando em $\lim _{t \rightarrow \infty}\langle Q, X(t)\rangle=0$, e a partir da definição 9 vem que $\mathcal{J}(X)=0$ (independente de $X$ ). Isto confirma o teorema 6. A figura 3.1 ilustra o custo de horizonte finito $J(t, X)$ para diferentes valores de $X$, e mostra, para $\bar{J}=1 / 3$, que $J(t, X)<t \bar{J}$ quando $t>t_{0}$, com $t_{0}$ que depende de $X$, veja proposição 6 . É interessante observar que este caso não é observável. 


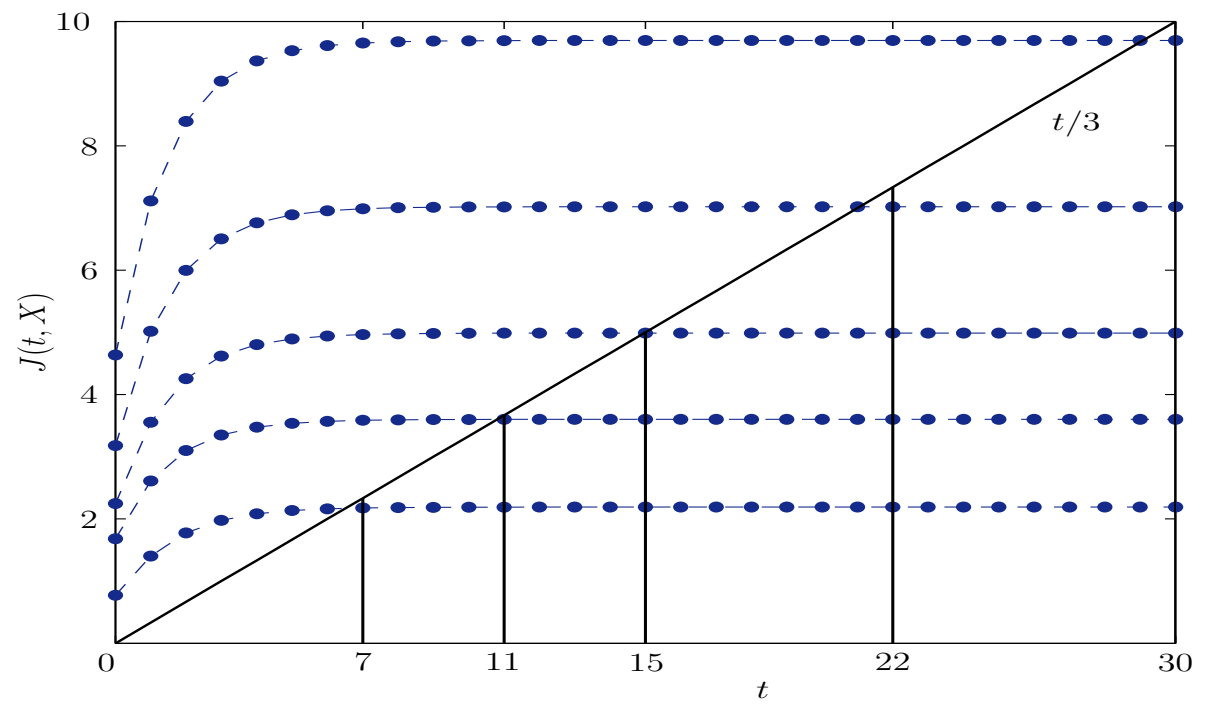

Figura 3.1: Custo $J(t, X)$ para 5 valores diferentes de $X$ (tracejado), a reta $t / 3$ (sólida) e os correspondentes valores de $t_{0}$, para o sistema no Caso 1.

Caso 2) ( $\alpha=0$ e $\beta=1 / 4$ ) Este caso é não assintoticamente estável no sentido de Lagrange, mas é fracamente detetável. A figura 3.2 ilustra $J(t, X)$ em escala logarítmica para diferentes valores de $X$, sugerindo que o custo médio a longo prazo não existe, o que está de acordo com o teorema 6 .

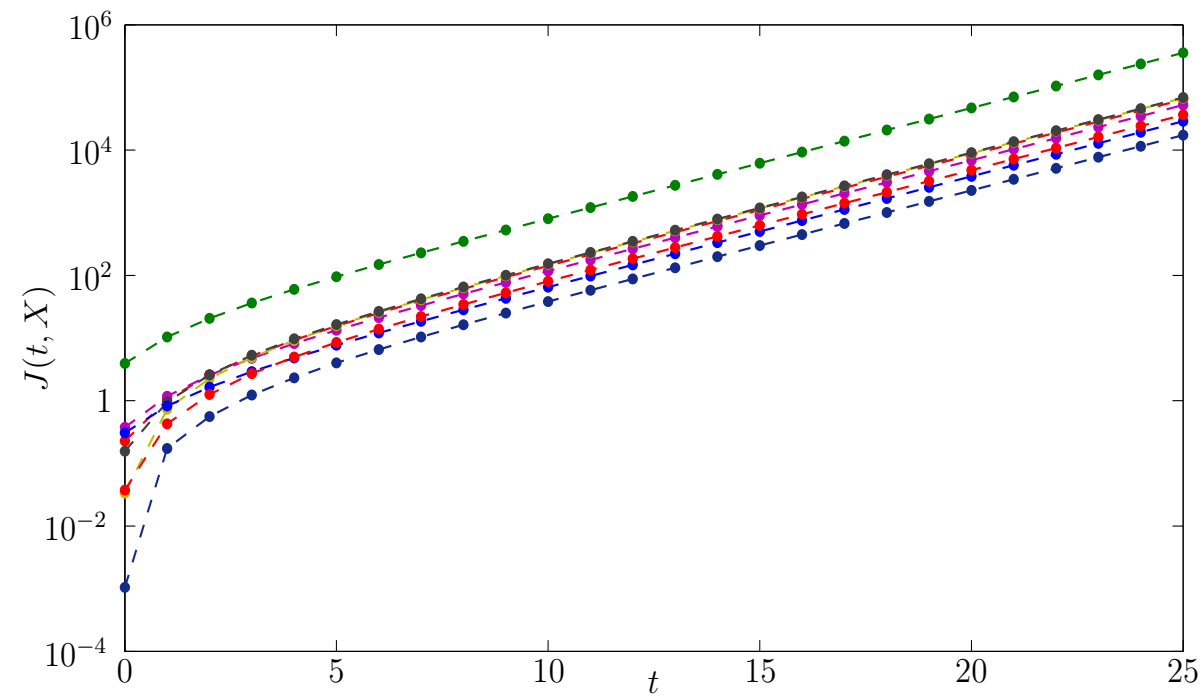

Figura 3.2: Custo $J(t, X)$ em escala logarítmica para 10 valores diferentes de $X$, para o sistema no Caso 2

Caso 3) ( $\alpha=0$ e $\beta=1 / 2$ ). O sistema não é assintoticamente estável no sentido Lagrange e é fracamente detetável. Nesta situação nós temos $\mathcal{T}(X)=\left(X_{2}, X_{2}\right)$, o que leva a $\langle Q, X(k)\rangle \geq X_{2}$ e $J(t, X)=\sum_{k}\langle Q, X(k)\rangle \geq k X_{2}$, e portanto $\mathcal{J} \geq X_{2}$ e o custo médio a longo prazo é não limitado de acordo com a definição 9. Neste caso é possível verificar que o custo médio a longo prazo existe para cada $X$, veja figura 3.3 . Isto mostra que a existência de $\mathcal{J}$ não implica na estabilidade, mesmo para sistemas detetáveis. 


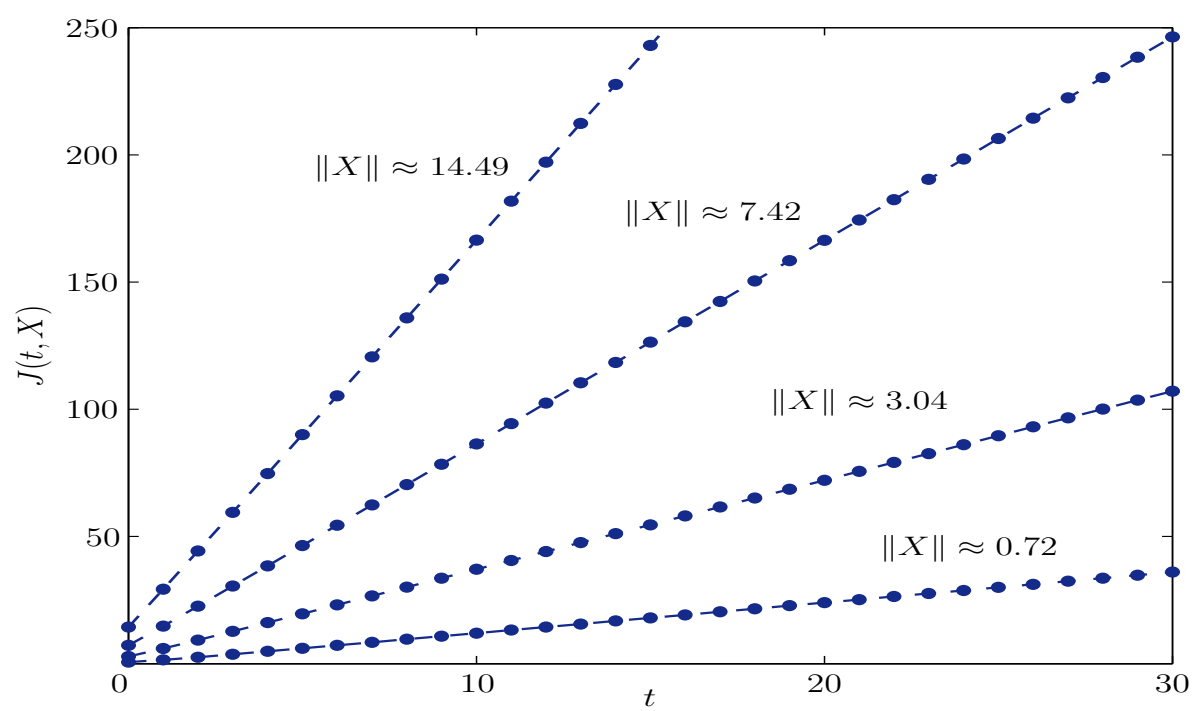

Figura 3.3: Custo $J(t, X)$ para diferentes valores de $X$, para o sistema no Caso 3.

Caso 4) ( $\alpha=1$ e $\beta=0$ ) Neste exemplo o sistema não é assintoticamente estável no sentido de Lagrange e não é detetável. O custo neste caso satisfaz $J(t, X)=\langle Q, X\rangle+t \pi_{1}(0)$, e portanto $\mathcal{J}=\pi_{1}(0) \leq 1$. Isto mostra que a limitação do custo não é condição suficiente para a estabilidade assintótica no sentido de Lagrange.

Note que não foi necessário, no exemplo acima, tomar $x$ com dimensão maior que 1 para ilustrar os diversos cenários possíveis. Por curiosidade, apresentamos abaixo um sistema detetável não observável com $x \in \mathbb{R}^{2}$ com custo médio a longo prazo limitado e assintoticamente estável no sentido de Lagrange que está de acordo com o teorema.

$$
\begin{gathered}
A_{1}=\left[\begin{array}{cc}
1 & 2 \\
-1 & -2
\end{array}\right] \quad A_{2}=\left[\begin{array}{cc}
0.5 & 0.5 \\
-0.5 & -0.5
\end{array}\right] \\
G_{1}=\left[\begin{array}{ll}
1 & 2 \\
2 & 4
\end{array}\right] \quad G_{2}=\left[\begin{array}{ll}
1 & 0 \\
0 & 1
\end{array}\right] \\
Q_{1}=\left[\begin{array}{ll}
1 & 1 \\
1 & 1
\end{array}\right] \quad Q_{2}=\left[\begin{array}{ll}
1 & 1 \\
1 & 1
\end{array}\right] \\
\mathbf{P}=\left[\begin{array}{ll}
0.5 & 0.5 \\
0.5 & 0.5
\end{array}\right]
\end{gathered}
$$

Para concluir esta seção vamos sintetizar o que foi constatado. Observamos no caso 1 que a observabilidade não é uma condição necessária para obter a estabilidade. No caso 2 vimos que detetabilidade apenas não é suficiente. No caso 3 vimos que a existência do custo não pode substituir a sua limitação e no caso 4 vimos que apenas custo limitado não é suficiente. Com isto concluímos que a limitação do custo médio a longo prazo e a detetabilidade fraca são condições independentes no sentido que nenhuma implica a outra. Por fim, vimos que o resultado se mantém válido para dimensão de $x$ maior que 1 . 


\subsection{Sistemas dinâmicos generalizados}

\subsubsection{Teorema Principal}

Suponha que o sistema $(M, \mathbb{N}, A, S)$ e o custo $W$ associado como na seção 2.2 satisfaçam as hipóteses $\mathrm{H} 1$ a $\mathrm{H} 4$ e H1' e que além disso o sistema seja fracamente detetável conforme definição 13 . Dada uma trajetória $x$ iniciada no ponto $a$ a cada instante no movimento a continuação do movimento, deve ser outro movimento que deve satisfazer a condição de detetabilidade também, portanto a cada instante de tempo o movimento deve satisfazer a condição de detetabilidade. Vamos acompanhar um movimento e anotar os instantes em que ele satisfaz a condição de contratividade e no caso em que ele não satisfizer, vamos anotar os instantes em que o custo é da ordem de $d(x(n, a), e)$. Vamos construir formalmente estes instantes.

Definimos os conjuntos $S_{n}(x, b), a \in M, x \in S$ recursivamente da seguinte forma

$$
\begin{aligned}
& S_{0}(x, a)= \begin{cases}(s, 1), & \text { se } d(x(s, a), e)<\delta d(x(0, a), e) \\
(t, 0), & \text { se } \quad d(x(s, a), e) \geq \delta d(x(0, a), e)\end{cases} \\
& S_{n+1}(x, a)=\left\{\begin{array}{l}
S_{n}(x, a) \cup\{(m+s, 1)\} \quad \text { se } \quad d(x(m+s, a), e)<\delta d(x(m, a), e) \\
S_{n}(x, a) \cup\{(m+t, 0)\} \quad \text { se } \quad d(x(m+s, a), e) \geq \delta d(x(m, a), a),
\end{array}\right.
\end{aligned}
$$

onde $m:=\max \left\{p \in \mathbb{N}:(p, 1)\right.$ or $\left.(p, 0) \in S_{n}(x, a)\right\}$. Definimos $S(x, a):=\bigcup_{n=0}^{\infty} S_{n}(x, a) \mathrm{O}$ conjunto $S(x, a)$ leva em consideração os instantes para os quais o sistema, com condição inicial $b$ e executando o movimento $x$, contrai de acordo com a condição de detetabilidade, os elementos da forma $(\cdot, 1)$, e os instantes em que o sistema "paga o custo", os elementos $(\cdot, 0)$. A partir destes conjuntos definimos os seguintes conjuntos que serão utilizados sistematicamente.

$$
\begin{aligned}
T_{n}(x, a) & =\{p \in \mathbb{N}:(p, 1) \text { or }(p, 0) \in S(x, a) \text { e } p \leq n\}, \\
C_{n}(x, a) & =\{p \in \mathbb{N}:(p, 1) \in S(x, a) \text { e } p \leq n\}, \\
N C_{n}(x, a) & =T_{n}(a) \backslash C_{n}(a)=\{p \in \mathbb{N}:(p, 0) \in S(x, a) \text { e } p \leq n\},
\end{aligned}
$$

temos que $T_{n}(x, a)=C_{n}(x, a) \cup N C_{n}(x, a)$. Dado um conjunto $B$ vamos denotar por $|B|$ sua cardinalidade. Observe que

$$
\left\lfloor\frac{n}{\max (s, t)}\right\rfloor \leq\left|T_{n}(x, a)\right| \leq\left\lfloor\frac{n}{\min (s, t)}\right\rfloor .
$$

Para mostrar que um sistema dinâmico com custo associado que satisfaz as hipóteses $H 1$ à $H_{4}$ e $H_{1}$ ' e é detetável deve ser assintoticamente estável no sentido de Lagrange vamos empregar três lemas. O primeiro destes lemas diz que é possível limitar o crescimento do movimento de acordo com cardinalidade dos conjuntos definidos acima. O segundo lema informa que devido a propriedade aditiva do custo é possível encontrar um limitante inferior para o custo baseado na cardinalidade de $N C$. O terceiro lema nos diz que há uma bola que quando visitada uma vez deve ser visita outra vez antes de um certo intervalo de 
tempo tenha decorrido, esse intervalo não depende do movimento ou da condição inicial. O teorema finaliza dizendo que esta bola é visitada seja qual for o movimento e a condição inicial.

Lema 1. Para cada $n \in \mathbb{N}$ e $a \in M$

$$
d(x(n, a), e) \leq L^{s+t\left|N C_{n}(x, a)\right|} \delta^{\left|C_{n}(x, a)\right|} d(a, e)
$$

Demonstração. Definimos $T(x, a):=\bigcup_{n=0}^{\infty} T_{n}(x, a)$. Vamos denotar os elementos $T(x, a)$ de acordo coma a ordem induzida de $\mathbb{N}$, ou seja, $t_{0}$ é o mínimo, $t_{1}$ é o mínimo excluindo $t_{0}$ e assim por diante. Vamos provar por indução em $n$ que

$$
d\left(x\left(t_{n}, a\right), e\right) \leq L^{t\left|N C_{t_{n}}(x, a)\right|} \delta^{\left|C_{t_{n}}(x, a)\right|} d(a, e) .
$$

Começamos com $n=0$, então

$$
d\left(x\left(t_{0}, a\right), e\right) \leq\left\{\begin{array}{l}
L^{t} d(a, e)=L^{t\left|C_{t_{0}}(x, a)\right|} d(a, e) \quad \text { se } \quad\left|N C_{t_{0}}(x, a)\right|=1 \\
\delta d(a, e)=\delta^{\left|C_{t_{0}}(x, a)\right|} d(a, e) \quad \text { se } \quad\left|C_{t_{0}}(x, a)\right|=1
\end{array} .\right.
$$

A primeira desigualdade nos diz que se $t_{0}$ não é um instante de contração então o movimento cresce no máximo $L^{t}$ vezes. Claramente temos que

$$
d\left(x\left(t_{0}, a\right), e\right) \leq L^{t\left|N C_{t_{0}}(x, a)\right|} \delta^{\left|C_{t_{0}}(x, a)\right|} d(a, e) .
$$

Assumindo (3.13) é válido para $n$ segue que

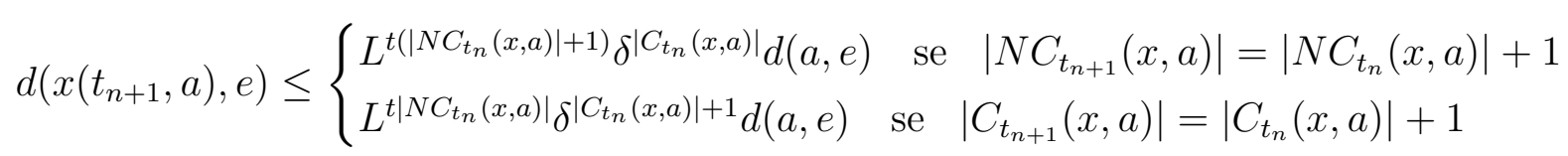

que é equivalente a

$$
d\left(x\left(t_{n+1}, a\right), e\right) \leq L^{t\left|N C_{t_{n+1}}(x, a)\right|} \delta^{\left|C_{t_{n+1}}(x, a)\right|} d(a, e) .
$$

Finalmente verificamos 3.12). Seja $k \in \mathbb{N}$ e $t_{n} \leq k<t_{n+1}$. Se $t_{n+1} \in \bigcup_{n=0}^{\infty} N C_{n}(x, a)$ então o limitante assegura que $d(x(k, a), e) \leq L^{t\left|N C_{k}(x, a)\right|} \delta^{\left|C_{k}(x, a)\right|} d(a, e)$. Se $t_{n+1} \in \bigcup_{n=0}^{\infty} C_{n}(x, a)$ então para garantir a limitação devemos multiplicar o limitante anterior por $L t_{n+1}-t_{n}$ vezes, ou seja, $s$ vezes resultando em $d(x(k, a), e) \leq L^{s+t\left|N C_{k}(x, a)\right|} \delta^{\left|C_{k}(x, a)\right|} d(a, e)$. Note que utilizamos aqui $C_{k}=C_{t_{n}}$ e $N C_{k}=N C_{t_{n}}$ para $k$ neste intervalo.

Observamos que para este lema foram usados a detetabilidade fraca do sistema e as hipóteses $\mathrm{H}_{3}$ e $\mathrm{H}_{4}$.

Lema 2. Para cada $a \in M$ e $n \in \mathbb{N}$

$$
W(n, x, a) \geq \gamma \sum_{i \in N C_{n}(x, a)} d(x(i-t, a), e)
$$


Demonstração. Seja $n c_{0}$ o mínimo de $N C_{n}(x, a), n c_{1}$ o mínimo excluindo $n c_{0}$ assim por diante. Temos que

$\sum_{i=0}^{n-1} x(i, a)=\sum_{i=0}^{n c_{0}-t-1} c(x(i, a))+\sum_{i=n c_{0}-t}^{n c_{0}-1} c(x(i, a))+\sum_{i=n c_{0}}^{n c_{1}-t-1} c(x(i, a))+\sum_{i=n c_{1}-t}^{n c_{1}-1} c(x(i, a))+\ldots$

onde nós colocamos $\sum_{i=a}^{b}=0$ se $b<a . W(n, x, b) \geq \sum_{i \in N C_{n}(x, a)} W(t-1, x(i-t+\cdot), a)$ e pela não contratividade destes instantes, nós temos (3.14).

Foram utilizadas as hipóteses de detetabilidade, H3 e a forma aditiva do custo.

Lema 3. Seja $B:=(W+1) \max (s, t)\left(1-t \log _{\delta} L\right) / \gamma$. Suponha que $L \geq 1$ em $H_{4} e$ $B<d(a, e) \leq L B$. Então existe $\bar{n}$, independente de a e do movimento $x$, e $n n<\bar{n}$ tal que $d(x(n, a), e) \leq B$.

Demonstração. Suponha que exista um par $(x, a)$ que satisfaz a seguinte condição

$$
d(x(n, a), e)>B
$$

para todo $n \leq \bar{n}$, com

$$
\left.\bar{n}=\max \left(\left\lceil g(L B)-\gamma B \frac{s+1}{1-t \log _{\delta} L}-(W+1) \max (s, t)\right\rceil\right), 1\right)
$$

então

$$
L^{s+t\left|N C_{n}(x, a)\right|} \delta^{\left|C_{n}(x, a)\right|} d(a, e) L>1 .
$$

Usando $C_{n}(x, a)=T_{n}(x, a)-N C_{n}(x, a)$ e resolvendo a inequação em $N C_{n}(x, a)$

$$
\left|N C_{n}(x, a)\right|>\frac{1}{1-t \log _{\delta} L}\left|T_{n}(x, a)\right|+\frac{s+1}{1-t \log _{\delta} L} .
$$

Aplicando o lema (3.14 e a hipótese H1')

$$
\begin{aligned}
W n+g(L B) & \geq W n+g(d(a, e)) \geq W(n, x, a) \geq \gamma \sum_{i \in N C_{n}(x, a)} d(x(i-t, a), e) \geq \\
& \geq \gamma B\left|N C_{n}(x, a)\right|>\gamma B \frac{1}{1-t \log _{\delta} L}\left|T_{n}(x, a)\right|+\gamma B \frac{s+1}{1-t \log _{\delta} L} \geq \\
& \geq(W+1) \max (s, t)\left\lfloor\frac{n}{\max (s, t)}\right\rfloor+\gamma B \frac{s+1}{1-t \log _{\delta} L} \geq \\
& \geq(W+1) n+\gamma B \frac{s+1}{1-t \log _{\delta} L}-(W+1) \max (s, t),
\end{aligned}
$$

portanto

$$
n<g(L B)-\gamma B \frac{s+1}{1-t \log _{\delta} L}-(W+1) \max (s, t) .
$$

Para $n=\bar{n}$ obtemos uma contradição. Segue que existe $n<\bar{n}$ tal que $d(x(n, b), a) \leq$ $B$. 
Observe que no lema anterior poderíamos escolher $a=y(n, b)$ e $x=y(n+\cdot, b)$, isto implica que se existe um instante de tempo tal que o movimento diste menos que $B$ do ponto de equilíbrio $e$ então o sistema não pode distar $L^{\bar{n}+1} B$ de $e$ nos instantes posteriores, isto é, o movimento fica "preso" nas proximidades de $e$. Vamos elaborar esta observação no próximo teorema.

Teorema 7. Suponha que um sistema dinâmico $(M, \mathbb{N}, A, S)$ com custo associado satisfaçam as hipóteses $\mathrm{H}_{1}$ a $\mathrm{H}_{4}$ e H1', se este sistema dinâmico generalizado for fracamente detetável então o sistema é assintoticamente estável no sentido de Lagrange.

Demonstração. Seja $B$ e barn como no lema anterior. Vamos assumir que $L \geq 1$ pois se esse não for o caso $d(x(n, a), e) \leq L^{n} d(a, e)$ e portanto $d(x(n, a), e) \leq L^{\bar{n}+1} B$ para $n$ suficientemente grande. Suponha que exista $x$ tal que

$$
d(x(n, a), e)>B L
$$

para todo $n$. Segue utilizando o lema 1 que

$$
L^{s+t\left|N C_{n}(x, a)\right|} \delta^{\left|C_{n}(x, a)\right|} d(a, e)>B L>B
$$

para todo $n$. Resolvendo a desigualdade para $N C_{n}(x, a)$ como no lema 3

$$
\left|N C_{n}(x, a)\right|>\frac{1}{1-t \log _{\delta} L}\left|T_{n}(x, a)\right|+\frac{\log _{\delta}\left(L^{s} B^{-1} d(a, e)\right)}{1-t \log _{\delta} L} .
$$

Utilizando o mesmo argumento que no lema anterior

$$
\begin{aligned}
W n+g(d(a, e)) & \geq W(n, x, a) \geq \gamma \sum_{i \in N C_{n}(x, a)} d(x(i-t, a), e) \geq \\
& \geq \gamma B\left|N C_{n}(x, a)\right|>\gamma B \frac{1}{1-t \log _{\delta} L}\left|T_{n}(x, a)\right|+\gamma B \frac{\log _{\delta}\left(L^{s} B^{-1} d(a, e)\right)}{1-t \log _{\delta} L} \geq \\
& \geq(W+1) \max (s, t)\left\lfloor\frac{n}{\max (s, t)}\right\rfloor+\gamma B \frac{\log _{\delta}\left(L^{s} B^{-1} d(a, e)\right)}{1-t \log _{\delta} L} \geq \\
& \geq(W+1) n+\gamma B \frac{\log _{\delta}\left(L^{s} B^{-1} d(a, e)\right)}{1-t \log _{\delta} L}-(W+1) \max (s, t)
\end{aligned}
$$

seja

segue que

$$
\alpha(a):=\gamma B \gamma B \frac{\log _{\delta}\left(L^{s} B^{-1} d(a, e)\right)}{1-t \log _{\delta} L}-(W+1) \max (s, t)-g(d(a, e))
$$

$$
W>W+1+\frac{\alpha(a)}{n}
$$

que é uma contradição para $n$ suficientemente grande pois $\lim _{n \rightarrow \infty} \alpha(a) / n=0$. Portanto deve existir $n(a)$ tal que $d(x(n, a), e) \leq B L$. Agora observe que podemos ter $d(x(n, a), e)>B$ ou $d(x(n, a), e) \leq B$. Se $d(x(n, a), e)>B$ o lema 3 implica que existe um instante $\hat{n}(a)<n(a)+\bar{n}$ tal que $d(x(n, a), e) \leq B)$. Devemos ter $d(x(n, a), e) \leq L^{\bar{n}+1} B$ entre $n(a)$ e $\hat{n}(a)$. Se $d(x(n, a), e) \leq B$, então podem ocorrer duas coisas, $d(x(n, a), e)<B$ ou $L \geq d(x(n+1, a), e)>B$, caso em que como vimos. Em qualquer caso temos $d(x(n, a), e) \leq L^{\bar{n}+1}$ para $n>n(a)$, o que prova o resultado. 


\subsubsection{Um resultado sobre estabilidade exponencial}

A intenção desta seção é mostrar que se $g(x)=\alpha d(x, e)$ na hipótese H1', a taxa de decrescimento da distância do movimento até a bola da raio $B$ é exponencial, enquanto o movimento não atingiu a bola.

Proposição 8. Se $d(a, e)>B$ então existe $\beta$ e $0 \geq \xi<1$ tal que $d(x(n, a), e)<$ $\beta \xi^{n} d(a, e)$, para $d(x(n, a), e)>B$.

Demonstração. Vamos calcular o máximo intervalo de tempo em que para qualquer $x$ e $a \operatorname{com} d(a, e)>B$

$$
d(x(n, a), e)>\delta d(a, e) .
$$

Utilizando o lema 1

$$
L^{s+t\left|N C_{n}(x, a)\right|} \delta^{\left|C_{n}(x, a)\right|}>\delta .
$$

Isso implica que

$$
W n+\alpha d(a, e)>\gamma \delta d(a, e)\left(\frac{1}{1-t \log _{\delta} L}\left|T_{n}(x, a)\right|-\frac{1}{1-t \log _{\delta} L}\right)
$$

que implica em

$$
W n>B\left(\gamma \delta \frac{1}{1-t \log _{\delta} L}\left\lfloor\frac{n}{\max (s, t)}\right\rfloor-\alpha-\gamma \delta \frac{1}{1-t \log _{\delta} L}\right)
$$

Como no lema 3 existe um $\bar{n}$ que não depende de $a$ e $x$ tal que existe um $n_{1}<\bar{n}$ com $d\left(x\left(n_{1}, a\right), e\right)<\delta d(a, e)$. Iniciando o movimento em $n_{1}$ e supondo que $d\left(x\left(n_{1}, a\right), e\right)>B$ podemos aplicar novamente o raciocínio acima e encontramos outro $n_{2}$ que satisfaz $d\left(x\left(n_{2}, a\right), e\right)<\delta d\left(x\left(n_{1}, a\right), e\right)$. Construímos assim uma sequência $n_{1}, n_{2}, \ldots, n_{i}$ com $n_{i+1}-n_{i} \geq \bar{n}$ tal que $d\left(x\left(n_{i+1}, a\right), e\right)<\delta d\left(x\left(n_{i}, e\right)\right.$ que é válida enquanto $d\left(x\left(n_{i}, e\right)>B\right.$. Entre $n_{i}$ e $n_{i+1}$ o movimento só pode crescer $L^{\bar{n}}$, segue que $d(x(n, a), e)<\delta^{i} d(a, e)$ com $n$ com $n_{i} \leq n \leq n_{i+1}$. Observe que

$$
\frac{n_{i}}{\bar{n}} \leq \frac{n}{\bar{n}} \leq \frac{n_{i}}{\bar{n}}+1 \Rightarrow \frac{n}{\bar{n}} \leq i+1
$$

pois $n_{i} \leq \bar{n} i$. Logo

$$
d(x(n, a), e)<L^{\bar{n}} \delta^{-1} \delta^{\frac{n}{\bar{n}}} d(a, e)
$$

sempre que $d(x(n, a), e)>B$. 


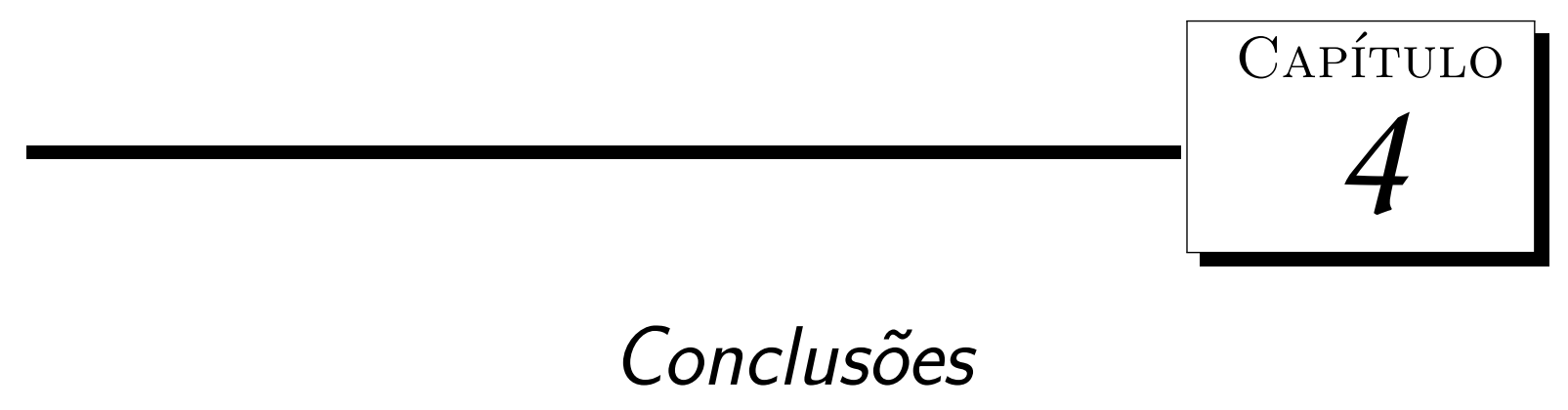

Neste trabalho foram estudadas condições suficientes para atingir a estabilidade assintótica no sentido de Lagrange em sistemas fracamente detetáveis. Para o caso de sistemas lineares com saltos markovianos fracamente detetáveis observamos que a condição de limitação do custo médio a longo prazo é necessária e também suficiente para a estabilidade, enquanto que no caso de sistemas dinâmicos generalizados obtemos uma condição mais forte do que a limitação do custo médio a longo prazo que garante a estabilidade de uma classe especial de sistemas.

Para sistemas lineares com saltos markovianos vimos através dos exemplos da subseção 3.1 .2 que a existência do custo médio a longo não pode substituir sua limitação, como no caso 3 da subseção. Vimos também que a observabilidade, que é mais forte que a detetabilidade, não é necessária para garantir estabilidade como no caso 1, que o custo médio ser limitado não implica em estabilidade como no caso 4, e que a detetabilidade não implica em estabilidade como no caso 2.

Por fim vimos que em um sistema com controle de realimentação por saída, com uma hipótese de detetabilidade fraca sobre a versão com controle nulo, a condição de estabilidade é equivalente a existência do custo médio a longo prazo. Em particular, se tomarmos um sistema com controle nulo fracamente detetável, e em seguida projetarmos um controle de realimentação de saída que estabilize o sistema no sentido em questão, então o custo médio a longo prazo sempre existe e portanto podemos utilizá-lo como um índice de desempenho deste sistema.

Já para sistemas dinâmicos generalizados fracamente detetáveis, vimos que se o custo associado ao sistema é limitado linearmente no tempo com condições especiais sobre o coeficiente linear, veja hipótese H1' da seção 2.2, então o sistema é assintoticamente estável no sentido de Lagrange se o sistema satisfaz alguma hipóteses. Como foi observado no fim da seção 2.2 esta classe de sistemas contém alguns casos bastante utilizados em modelagem. Vimos, enfim, que a distância entre um ponto qualquer e a bola de raio $r$ centrada em e especificada na definição 14 decresce exponencialmente no caso em que o 
coeficiente linear, da hipótese H1', é linear na distância, veja subseção 3.2.2. 


\section{Referências Bibliográficas}

Abramovich, Y.; Aliprantis, C. An invitation to operator theory. Graduate studies in mathematics. American Mathematical Society, 2002.

Aliprantis, C.; Burkinshaw, O. Positive operators. Pure and applied mathematics. Springer, 2006.

Aliprantis, C.; Tourky, R. Cones and duality. Graduate studies in mathematics. American Mathematical Society, 2007.

Anderson, B. D. O.; Moore, J. B. Detectability and stabilizability of time-varying discrete-time linear systems. SIAM Journal on Control and Optimization, v. 19, n. 1, p. 20-32, 1981.

Barbosa, B. G.; Costa, E. F. Lagrange asymptotic stability of weak detectable markov jump linear systems with bounded long run average cost. submetido, 2012a.

Barbosa, B. G.; Costa, E. F. On the stability of weakly observable Markov jump linear systems with bounded long run average cost. In: aguardando publicação, 2012b.

Boyd, S.; Vandenberghe, L. Convex optimization. Cambridge University Press, 2004.

Bueno, H. Álgebra linear: Um segundo curso. 1 ed. Sociedade Brasileira de Matemàtica, 2006.

Chung, K. A course in probability theory. Academic Press, 2001.

Cinlar, E. Introduction to stochastic processes. 1 ed. Prentice Hall College Div, 1997.

Coelho, F.; Lourenço, L. Um curso de Álgebra linear. 2 ed. Editora da Universidade de São Paulo, 2010.

Costa, E. F.; Do VAL, J. B. R. On the detectability and observability for discretetime Markov jump linear systems. System and Control Letters, v. 44, n. 2, p. 135-145, 2001. 
Costa, E. F.; Do VAL, J. B. R. Weak detectability and the linear-quadratic control problem of discrete-time markov jump linear systems. International Journal of Control, v. 75, n. 16-17, p. 1282-1292, 2002.

Costa, E. F.; DO VAL, J. B. Optimal cost convergence with respect to the time horizon. In: Proceedings of the European Control Conference 2003, 2003.

Costa, E. F.; Vargas, A. N.; Do VAL, J. B. R. Quadratic costs and second moments of jump linear systems with general Markov chain. Mathematics of Control, Signals, and Systems (MCSS), v. 23, p. 141-157, 2011.

Costa, O. L. V.; Fragoso, M. D.; Marques, R. P. Discrete-time Markov jump linear systems. 1 ed. Springer-Verlag, 2005.

Damm, T. On detectability of stochastic systems. Automatica, v. 43, n. 5, p. $928-$ 933, 2007.

Damm, T.; Ethington, C. Detectability, observability, and asymptotic reconstructability of positive systems. In: Bru, R.; Romero-Vivó, S., eds. Positive Systems, v. 389 de Lecture Notes in Control and Information Sciences, Springer Berlin / Heidelberg, p. 63-70, 2009.

Deimling, K. Nonlinear functional analysis. Springer-Verlag, 1985.

Drăgan, V.; Morozan, T.; Stoica, A. M. Mathematical methods in robust control of discrete-time linear stochastic systems. Springer, 2009.

Hahn, W.; BaArtz, A. Stability of motion. Grundlehren der mathematischen Wissenschaften. Springer, 1967.

Kallenberg, O. Foundations of modern probability. Probability and its applications. Springer, 2002.

Kalman, R. Contributions to the theory of optimal control. 1960.

Karlin, S.; TAYlor, H. M. A first course in stochastic processes. 2 ed. Academic Press, 1975.

Kozin, F. A survey of stability of stochastic systems. Automatica, v. 5, n. 1, p. $95-$ $112,1969$.

Krasnoselskit, M.; Lifshits, E.; Sobolev, A. Positive linear systems: the method of positive operators. Sigma series in applied mathematics. Heldermann, 1989.

Li, Z.-Y.; Wang, Y.; Zhou, B.; Duan, G.-R. Detectability and observability of discrete-time stochastic systems and their applications. Automatica, v. 45, n. 5, p. $1340-1346,2009$.

Liao, X.; Wang, L.; Yu, P.; Yu, P. Stability of dynamical systems. Monograph series on nonlinear science and complexity. Elsevier, 2007. 
Lima, E. Álgebra linear. 7 ed. Instituto Nacional de Matemática Pura e Aplicada, 2004.

Michel, A.; Hou, L.; LiU, D. Stability of dynamical systems: continuous, discontinuous, and discrete systems. Systems \& control. Birkhaüser, 2008.

Michel, A.; WAng, K.; Hu, B. Qualitative theory of dynamical systems: the role of stability preserving mappings. Pure and applied mathematics. M. Dekker, 2001.

Norris, J. R. Markov chains. 1 ed. Cambridge University Pres, 1998.

Schaefer, H.; Wolff, M. Topological vector spaces. Graduate texts in mathematics. Springer, 1999.

Shiriatv, A. Probability. Graduate texts in mathematics. Springer, 1996.

Silva, C. A.; Costa, E. F. An algorithm for the long run average cost problem for linear systems with non-observed Markov jump parameters. In: In Proceedings of the 2009 American Control Conference, 2009, p. 4434 -4439.

Vargas, A.; Costa, E.; Do Val, J. B. Bounds for the finite horizon cost of Markov jump linear systems with additive noise and convergence for the long run average cost. In: Decision and Control, 2006 45th IEEE Conference on, 2006, p. $5543-5548$.

VArgas, A. N.; DO VAL, J. Stationary policies for the second moment stability in a class of stochastic systems. In: Joint 50th IEEE Conference on Decision and Control and European Control Conference, 2011, p. 1264-1268.

VArgas, A. N.; do VAL, J. B. R. Average cost and stability of time-varying linear systems. IEEE Transactions on Automatic Control, v. 55, n. 3, p. 714 -720, 2010.

Willems, J. Paradigms and puzzles in the theory of dynamical systems. Automatic Control, IEEE Transactions on, v. 36, n. 3, p. 259 -294, 1991.

Zubov, V. Methods of a.m. lyapunov and their application. P. Noordhoff, 1964. 
APÊNDICE

\section{Apêndice}

\section{A.1 Cadeias de Markov}

A definição dada para cadeias de Markov no texto não é a padrão dos textos de processos estocásticos como Cinlar (1997), Karlin e Taylor (1975) ou Norris (1998). Vamos mostrar que as duas definições concordam.

Definição 15. Uma família de variáveis aleatórias $(\theta(k))_{k \in \mathbb{N}}$ forma uma cadeia de Markov do tipo 1 se

$$
P(\theta(k) \mid \theta(k-1), \ldots, \theta(0))=P(\theta(k) \mid \theta(k-1)) .
$$

Definição 16. Uma família de variáveis aleatórias $(\theta(k))_{k \in \mathbb{N}}$ forma uma cadeia de Markov do tipo 2 se $\theta(t), t>k$ é condicionalmente independente de $\theta(n), n<k$ dado $\theta(k)$.

A prova de equivalência segue Shiriaev (1996).

Proposição 9. Uma cadeia de Markov é do tipo 1 se e somente se é do tipo 2.

Demonstração. $(\Leftarrow)$ Definimos os conjuntos

$$
\begin{aligned}
& B_{k}=\left\{\theta(0)=i_{0}, \theta(1)=i_{1}, \ldots, \theta(k-1)=i_{k-1}\right\}, \\
& F_{k, n}=\left\{\theta(k+1)=i_{k+1}, \theta(k+2)=i_{k+2}, \ldots, \theta(n)=i_{n}\right\}, \\
& N_{k}=\left\{\theta(k)=i_{k}\right\},
\end{aligned}
$$

Os conjuntos $B_{k}, F_{k, n}, N_{k}$ representam o passado, futuro até o instante $n$ e presente em relação a $k$. O futuro é condicionalmente independente do passado dado o presente se e somente se o processo é do Markov do tipo 2 1 . Vamos realizar o seguinte cálculo

$$
\begin{aligned}
& P\left(N_{k+1} \cap B_{k} \mid N_{k}\right)=P\left(N_{k+1} \mid N_{k}\right) P\left(B_{k} \mid N_{k}\right) \\
& P\left(N_{k+1} \mid B_{k} \cap N_{k}\right) P\left(B_{k} \mid N_{k}\right)=P\left(N_{k+1} \mid N_{k}\right) P\left(B_{k} \mid N_{k}\right)
\end{aligned}
$$

\footnotetext{
${ }^{1} \mathrm{O}$ leitor pode apreciar este resultado em face do Corolário 3.7 de Kallenberg (2002), que é requer mais material do que o que dispomos aqui
} 
Supondo $P\left(B_{k} \mid N_{k}\right) \neq 0$, obtemos o resultado esperado. $P\left(B_{k} \mid N_{k}\right)=0$ é necessário mais materila que o disponível aqui, consulte (Chung, 2001, Teorema 9.2.4).

$(\Rightarrow)$ Supondo que o processo é Markov do tipo 1, então pela expressão

$$
P(I \cap J \mid K)=P(I \mid J \cap K) P(J \mid K)
$$

e um argumento de indução mostra-se que

$$
P\left(F_{k, n} \mid N_{k} \cap B_{k}\right)=P\left(F_{k, n} \mid N_{k}\right)
$$

Aplicando A.1 com $I=F_{k, n}, J=B_{k}, K=N_{k}$ vemos que obtemos a propriedade de Markov do tipo 2.

\section{A.2 Alguns resultados em Álgebra linear e séries}

Nesta seção estão presentes resultados de álgebra linear utilizados nas demonstrações dos teoremas e um resultado sobre singularidades de séries utilizda no teorema de KreinRutman 2.

Lema 4. Seja $T$ um operador linear. Se $\rho(T)<1$ então $\lim _{t \rightarrow \infty}\left\|T^{k}\right\|=0$.

Demonstração. Existe uma base no qual a matriz que representa o operador está na forma de Jordan. Potências deste operador são representadas por potências da matriz de Jordan. Vamos mostrar que a matriz de Jordan converge a zero. Temos que:

$$
J=\left[\begin{array}{ccccc}
J\left(r_{1}, \lambda_{1}\right) & 0 & 0 & \ldots & 0 \\
0 & J\left(r_{2}, \lambda_{2}\right) & 0 & \ldots & 0 \\
\vdots & \ldots & \ddots & \ldots & \vdots \\
0 & \ldots & 0 & J\left(r_{n-1}, \lambda_{n-1}\right) & 0 \\
0 & \ldots & \ldots & 0 & J\left(r_{n}, \lambda_{n}\right)
\end{array}\right]
$$

onde as matrizes $J(r, \lambda)$ são de ordem $r \times r$.

$$
J\left(r_{i}, \lambda\right)=\left[\begin{array}{ccccc}
\lambda & 1 & 0 & \cdots & 0 \\
0 & \lambda & 1 & \cdots & 0 \\
\vdots & \vdots & \ddots & \ddots & \vdots \\
0 & 0 & \cdots & \lambda & 1 \\
0 & 0 & \cdots & 0 & \lambda
\end{array}\right]
$$

Como $J$ é bloco diagonal,

$$
J=\left[\begin{array}{ccccc}
J\left(r_{1}, \lambda_{1}\right)^{t} & 0 & 0 & \ldots & 0 \\
0 & J\left(r_{2}, \lambda_{2}\right)^{t} & 0 & \ldots & 0 \\
\vdots & \ldots & \ddots & \ldots & \vdots \\
0 & \ldots & 0 & J\left(r_{n-1}, \lambda_{n-1}\right)^{t} & 0 \\
0 & \ldots & \ldots & 0 & J\left(r_{n}, \lambda_{n}\right)^{t}
\end{array}\right]
$$


O $t$-ésimo produto das matrizes para $t \geq r$ pode ser calculado como

$$
J\left(r, \lambda_{i}\right)^{t}=\left[\begin{array}{ccccc}
\lambda^{t} & \left(\begin{array}{c}
t \\
1
\end{array}\right) \lambda^{t-1} & \left(\begin{array}{c}
t \\
2
\end{array}\right) \lambda^{t-2} & \ldots & \left(\begin{array}{c}
t \\
r-1
\end{array}\right) \lambda^{t-r+1} \\
0 & \lambda^{t} & \left(\begin{array}{c}
t \\
1
\end{array}\right) \lambda^{t-1} & \ldots & \left(\begin{array}{c}
t \\
r-2
\end{array}\right) \lambda_{i}^{t-r+2} \\
\vdots & \vdots & \ddots & \ddots & \vdots \\
0 & 0 & \cdots & \lambda^{t} & \left(\begin{array}{c}
t \\
1
\end{array}\right) \lambda^{t-1} \\
0 & 0 & \cdots & 0 & \lambda^{t}
\end{array}\right]
$$

Então, se $\rho(T)<1$ então temos que $\left|\lambda_{i}\right|<1$ para todo $i$, de tal forma que

$$
\lim _{t \rightarrow \infty} J(r, \lambda)_{i j}^{t}=0
$$

o que implica

$$
\lim _{t \rightarrow \infty} J_{i j}^{t}=0 .
$$

Como as coordenadas da matriz $J^{t}$ tendem a zero quando $t \rightarrow \infty$ então em qualquer norma $\left\|J^{t}\right\|$ tende a zero. Portanto o operador $T$ é tal que $\left\|T^{t}\right\|$ tende a zero em qualquer norma incluindo a norma do supremo que estamos utilizando. Observe que nesta demonstração estamos utilizando os complexos como corpo base e que estamos trabalhando com o operador $T$ complexificado cuja "estabilidade" é equivalente ao de $T$ real.

Proposição 10. Seja T um operador. Para cada $\epsilon>0$ existe $t_{0}$ tal que para $t>t_{0}$,

$$
\left\|T^{t}\right\| \leq(\rho(T)+\epsilon)^{t}
$$

Demonstração. Defina o operador $\hat{T}=(\rho(T)+\epsilon)^{-1} T$. Como $\rho(\hat{T})<1$, pelo lema anterior temos que $\left\|\hat{T}^{t}\right\|<1$ para $t$ suficientemente grande. Isto é Existe $t_{0}$ tal que

$$
\left\|T^{t}\right\| \leq(\rho(T)+\epsilon)^{t}
$$

para $t>t_{0}$ como queríamos demonstrar.

O próximo teorema a ser apresentado é o teorema de espectral para matrizes simétricas reais, sua prova ser encontrada em (Bueno, 2006, Teorema 10.4), (Coelho e Lourenço, 2010, Corolário 7.4.9) ou (Lima, 2004, Teorema 13.6).

Teorema 8 (Teorema Espectral (operadores simétricos)). Seja A uma matriz simétrica. Então existe $U$ com $U^{\prime}=U^{-1}$ tal que $U^{\prime} A U$ é uma matriz diagonal.

Lembramos que o polinômio característico de uma matriz $A$ é definido como $p(\lambda):=$ $\operatorname{det}(A-\lambda I)$ onde $I$ é a matriz identidade. Quando utilizarmos a notação $p(A)$ queremos dizer que no lugar dos variável do polinômio estamos inserindo a matriz $A$. Para a prova do próximo teorema veja por exemplo (Bueno, 2006, Corolário 5.22), (Coelho e Lourenço, 2010, Seção 5.3) ou (Lima, 2004, Capítulo 21).

Teorema 9 (Cayley-Hamilton). Seja A uma matriz e $p(\lambda)$ seu polinômio característico. Então $p(A)=0$. 
Para finalizar a proposição 19.8 de Deimling (1985) para completar o teorema de Krein-Rutman.

Proposição 11. Suponha que $\phi(\lambda)=\sum_{i=0}^{\infty} a_{n} \lambda^{n}$ tenha raio de convergência $r>0$. Suponha também que $a_{n} \geq 0$ e que $a_{n}>0$ para infinitos valores de $n$. Então $r$ é uma singularidade de $\phi$.

Demonstração. Suponha que $r$ não seja singularidade. Então para algum $\mu<r$ suficientemente próximo a série

$$
\phi(\lambda)=\sum_{k=0}^{\infty} \frac{\phi^{(k)}(\mu)}{k !}(\lambda-\mu)^{k}
$$

converge para algum $\lambda>r$. Seja $\mu=\alpha r \operatorname{com} \alpha<1$ e $\lambda=r(1+\delta)$ Então

$$
\sum_{k=0}^{\infty}(1-\alpha+\delta)^{k} r^{k} \sum_{n=k}^{\infty} a_{n}(\alpha r)^{n-k}
$$

é convergente. Como todos os termos destas soma dupla são não negativos podemos trocar a ordem das somas levando a contradição que

$$
\sum_{n=0}^{\infty} a_{n}(1+\delta)^{n} r^{n}
$$

é convergente.

\section{A.3 Testes de estabilidade e detetabilidade para sistemas lineares com saltos Markovianos}

Considere o sistema $(A,, 0, \cdot, \mathbf{P})$. Definimos $\mathcal{A}:=\left(\mathbf{P}^{\prime} \otimes I_{n^{2}}\right) \operatorname{diag}\left[A_{i} \otimes A_{i}\right]$ onde $\otimes$ denota o produto de Kronecker, isto é

$$
A \otimes B=\left[\begin{array}{ccc}
a_{11} B & \ldots & a_{1 n} B \\
\vdots & \ddots & \vdots \\
a_{n 1} B & \ldots & a_{n n} B
\end{array}\right]
$$

e diag a matriz formada por entradas diagonais

$$
\operatorname{diag}\left(A_{i}\right)=\left[\begin{array}{ccccc}
A_{1} & \ldots 0_{n} \vdots & \ddots & \vdots \\
0_{n} & \ldots & A_{n} &
\end{array}\right]
$$

A próxima proposição fornece um método para verificar que $\rho(\mathcal{T})<1$. Para indicações da prova veja (Costa et al., 2005, Capítulo 3).

Proposição 12. $\rho(\mathcal{T})<1$ se e somente se $\rho(\mathcal{A})<1$. 
Os próximos dois testes dizem respeito a detetabilidade fraca. Considere as matrizes definidas recursivamente por. Considere a coleção d matrizes $O(t)$ definidas como

$$
O_{i}(t)=\sum_{i=0}^{t} \mathcal{T}_{i}^{*}(Q),
$$

onde $\mathcal{T}^{*}$ é o operador adjunto de $\mathcal{T}$ com respeito ao produto interno definido na seção 2.1. Explicitamente

$$
\mathcal{T}_{i}^{*}(X)=A_{i}^{\prime} \sum_{j=1}^{N} p_{i j} X_{j} A_{i} .
$$

Defina a coleção de matrizes $\mathcal{O}$ com

$$
\mathcal{O}=\left[O_{i}(0) O_{i}(1) \ldots O_{i}\left(N^{2} n-1\right)\right]
$$

O próximo teorema fornece uma forma de testar a detetabilidade fraca através de desigualdades matriciais lineares. Este teorema decorre imediatamente do teorema 1 de Costa e Do Val (2002) e da proposição 3.43 de Costa et al. (2005).

Teorema 10 (Teste de W-Detetabilidade). O sistema $(A, 0, Q, \mathbf{P})$ é fracamente detetável de se e somente se existem matrizes $U \geq 0 \in\left(\mathbb{M}^{n, n}\right)^{N}, V \in\left(\mathbb{M}^{n, s}\right)^{N}, W \geq 0 \in\left(\mathbb{M}^{s, s}\right)^{N} e$ $Z \geq 0 \in\left(\mathbb{M}^{n, n}\right)^{N}$ satisfazendo:

$$
\begin{gathered}
A_{i}^{\prime} Z_{i} A_{i}+\mathcal{O}_{i}^{\prime} V_{i}^{\prime} A_{i}+A_{i}^{\prime} V_{i} \mathcal{O}_{i}+C_{i}^{\prime} W_{i} C_{i}+U_{i}<0 \\
{\left[\begin{array}{cc}
Z_{i} & V_{i} \\
V_{i}^{\prime} & W_{i}
\end{array}\right] \geq 0} \\
Z_{i} \geq \mathcal{E}_{i}(U), U_{i}>0, Z_{i}>0
\end{gathered}
$$

para todo $i \in S$.

Outro teste presente na literatura é o teste de Hautus. Para uma prova para sistemas estocásticos sem saltos que pode ser adaptada veja Li et al. (2009).

Teorema 11. O sistema $(A, 0, G, \mathbf{P})$ é fracamente detetável se só se para todo autovetor $V \in S_{n}^{+}$de $\mathcal{T}$ com autovalor $\lambda$ que satisfaz $|\lambda| \geq 1$ tem-se $V Q \neq 0$. O sistema é fracamente observável se se para todo autovetor $V \in S_{n}^{+}$de $\mathcal{T} V Q \neq 0$. 